University of Nebraska - Lincoln

DigitalCommons@University of Nebraska - Lincoln

2012

\title{
Application of PDF mixing models to premixed flames with differential diffusion
}

\author{
Edward Richardson \\ University of Southampton, Southampton, UK \\ Jacqueline Chen \\ Sandia National Laboratories, jhchen@sandia.gov
}

Follow this and additional works at: https://digitalcommons.unl.edu/usdoepub

Part of the Bioresource and Agricultural Engineering Commons

Richardson, Edward and Chen, Jacqueline, "Application of PDF mixing models to premixed flames with differential diffusion" (2012). US Department of Energy Publications. 129.

https://digitalcommons.unl.edu/usdoepub/129

This Article is brought to you for free and open access by the U.S. Department of Energy at DigitalCommons@University of Nebraska - Lincoln. It has been accepted for inclusion in US Department of Energy Publications by an authorized administrator of DigitalCommons@University of Nebraska - Lincoln. 


\title{
Application of PDF mixing models to premixed flames with differential diffusion
}

\author{
Edward S. Richardson ${ }^{\mathrm{a}, *}$, Jacqueline H. Chen ${ }^{\mathrm{b}}$ \\ ${ }^{a}$ Faculty of Engineering and the Environment, University of Southampton, Southampton SO17 1BJ, UK \\ ${ }^{\mathrm{b}}$ Reacting Flow Research Department, Combustion Research Facility, Sandia National Laboratories, P.O. Box 969, MS 9051, Livermore, CA 94551-0969, United States
}

\section{A R T I C L E I N F O}

\section{Article history:}

Received 20 April 2010

Received in revised form 22 September 2011

Accepted 29 February 2012

Available online 23 March 2012

\section{Keywords:}

Probability density function

Mixing models

Differential diffusion

Direct numerical simulation

Premixed turbulent combustion

\begin{abstract}
A B S T R A C T
Differential diffusion alters the balance of reaction and diffusion in turbulent premixed combustion, affecting the performance and emissions of combustion devices. Modelling combustion devices with Probability or Filtered Density Function (PDF or FDF) methods provides an exact treatment for the change in composition due to chemical reaction, while molecular mixing has to be modelled. Previous PDF molecular mixing models do not account for differential diffusion in a manner which satisfies realizability requirements. A new approach for treating differential diffusion, which ensures realizability, is proposed for pairwise-exchange mixing models in general, and applied in the Interaction by Exchange with the Mean (IEM) model of Dopazo [26], and in the Euclidean Minimum Spanning Tree (EMST) model of Subramaniam and Pope [5]. The new differential diffusion models are referred to as IEM-DD and EMST-DD respectively.

Results from two and three-dimensional DNS of turbulent premixed methane-air combustion show that mixing rates and conditional statistics of species mass fractions depend on species diffusivities and the combustion regime. Zero-dimensional PDF model results obtained for the two-dimensional DNS case show that the EMST-DD model best reproduces the features that characterize differential diffusion in the DNS. The essential feature of the EMST-DD model, which accounts for its success in turbulent premixed combustion, is that differential mixing rates are imposed within a model which mixes locally in composition space.
\end{abstract}

Published by Elsevier Inc. on behalf of The Combustion Institute.

\section{Introduction}

Modelling of turbulent reacting flows requires closure for averaged or filtered chemical source terms. Probability density function (PDF) methods [1-3] provide an exact closure for the non-linear dependencies of reaction rates on the turbulent distribution of composition and temperature. The molecular mixing terms in transported PDF models, however, are unclosed and must be modelled. Under certain conditions, in the presence of flames, modelling for the effects of the molecular mixing on the composition PDF can be deficient [4]. Attempts have been made to address aspects of mixing associated with flames, such as localness (described later in this section) $[5,6]$ and differential diffusion $[7,8]$. The objective of this paper is to assess the capability of existing models to represent mixing in turbulent premixed flames, and to investigate modifications to enhance their treatment of differential diffusion.

The partial differential equation governing the mass fractions $Y_{\alpha}$ in a gas phase reacting system is: $\frac{\partial \rho Y_{\alpha}}{\partial t}+\frac{\partial \rho u_{i} Y_{\alpha}}{\partial x_{i}}=\frac{\partial J_{i}^{\alpha}}{\partial x_{i}}+\rho S_{\alpha}$

where $\rho$ is density, $J_{i}^{\alpha}$ is the diffusion flux of species $\alpha$ in the $i$ th direction, and $S_{\alpha}$ is the chemical source term. The usual summation convention applies to repeated Roman subscripts. The corresponding transport equation for the joint-scalar PDF, $f_{Y}$, is given by (Ref. [9] (Eq. (32))):

$$
\begin{aligned}
\frac{\partial \rho f_{Y}}{\partial t}+\frac{\partial \rho \tilde{u}_{i} f_{Y}}{\partial x_{i}}= & -\frac{\partial \rho S_{\alpha} f_{Y}}{\partial \psi_{\alpha}}-\frac{\partial}{\partial x_{i}}\left[\left\langle u_{i}^{\prime \prime} \mid \psi\right\rangle \rho f_{Y}\right] \\
& +\frac{\partial}{\partial \psi_{\alpha}}\left[\left\langle\frac{\partial J_{i}^{\alpha}}{\partial x_{i}} \mid \psi\right\rangle f_{Y}\right] .
\end{aligned}
$$

The three terms on the right hand side represent the contribution due to the chemical source term $S_{\alpha}$, the turbulent transport of the $\mathrm{PDF}$, and the conditional diffusion term involving transport across the scalar sample-space, $\psi$, due to the diffusion flux $J_{i}^{\alpha} \cdot\langle\cdot\rangle$ denotes an ensemble average, and $\langle\cdot \mid \psi\rangle$ is an average conditioned upon $\mathbf{Y}=\psi$. The tilde $\sim$ denotes a density weighted (Favre) average, and " indicates a Favre fluctuation. $t$ is time, $x_{i}$ and $u_{i}$ are components of position and velocity. The conditional diffusion term is unclosed and its contribution can be divided into two processes [9]: spatial transport by molecular diffusion; and local composition-space

\footnotetext{
* Corresponding author. Fax: +44023 80593230

E-mail address: e.s.richardson@soton.ac.uk (E.S. Richardson).
} 
mixing. Spatial transport by molecular diffusion can be significant in low Reynolds number turbulent mixing, or in Large Eddy Simulation (LES) with low filter-scale Reynolds numbers, and McDermott and Pope [10] present modelling of diffusive spatial transport including effects of differential diffusion. Modelling of the latter process - local composition-space mixing - is the subject of this study.

Joint-scalar PDF transport models for turbulent combustion are commonly implemented by representing the mixture statistics by a set of notional particles, each bearing a composition, enthalpy, and a statistical weighting. Modelling of the composition-space mixing typically involves two steps: (1) specification of a turbulent scalar dissipation rate and (2) application of a mixing model which uses the specified scalar dissipation rate.

The mean scalar dissipation rate, which is the rate of decrease of a scalar's variance resulting solely from molecular diffusion, is equal to $\widetilde{\chi_{\alpha}}=-2 \widetilde{Y}_{\alpha}^{\prime \prime} \Gamma_{\alpha}$ (from Eq. (B.2) in Ref. [9]), where $\Gamma_{\alpha}$ is the diffusion source term in Eq. (1): $\Gamma_{\alpha}=-\left(\partial J_{i}^{\alpha} / \partial x_{i}\right) / \rho$. Note that further simplification to the commonly used expression for scalar dissipation $\widetilde{\chi_{\alpha}}=2 D_{\alpha}\left(\nabla Y_{\alpha}^{\prime \prime}\right)^{2}$ requires assumptions of Fickian diffusion with equal diffusivities, and we do not make these assumptions in this study. In modelling of premixed combustion, the mean scalar dissipation rate of a reaction progress variable may be obtained from modelled transport equations [11-15] or algebraic relations applicable to a range of combustion regimes [14-16]. For their PDF model of premixed combustion, Lindstedt and Vaos [17] used a model by Kuan et al. [16] for progress variable dissipation to impose a single variance decay rate for all reactive scalars. DNS studies of the mixing rates in non-premixed combustion [18] and in premixed combustion [19] show, however, that wide discrepancies occur among species mixing rates due to the small-scale scalar gradients imposed on different species by the presence of flame structures. The occurrence of different mixing rates among the scalars is a feature of flamelet combustion that it is desirable for PDF mixing models to reproduce.

Subramaniam and Pope [5] provide a set of performance criteria for mixing models: conservation of means, decay of variances, boundedness, linearity and independence, relaxation to a Gaussian, dependence on Reynolds number (Re), which are relevant in all turbulent flows; and localness, treatment of differential diffusion, and dependence on length scales of scalar fields, which are especially pertinent to the discussion of premixed combustion in this paper.

In the flamelet regime of turbulent premixed combustion the scalar length scales, and hence the molecular diffusion rates of reactive scalars, are imposed predominantly by the reactiondiffusion balance in the flame front, rather than by the turbulence cascade process. It is observed from several DNS studies of mixing in homogeneous turbulence [20-24] that, when the scalar length scales are controlled by the turbulence cascade process, the mean scalar dissipation rate is independent of species diffusivities, and proportional to the turbulent frequency, $\omega=\epsilon / k$ (where $\epsilon$ is the dissipation rate of turbulent kinetic energy $k$ ). The simulations by Juneja and Pope [20] are initialized with a scalar field which is independent from the turbulent field. In this situation, they observe that the initial scalar dissipation rates depend on the length scales characterizing the scalar fields (shorter length scales increase the scalar dissipation rate), and on the species diffusivities (higher diffusivities increase the scalar dissipation rate). Because, in the flamelet regime, the scalar length scales are not controlled only by the turbulence cascade process, we expect the mean scalar dissipation rate to depend partly on the flame length scales, and on the molecular diffusivities of the individual scalars. The transition between flamelet premixed combustion and distributed premixed combustion is delineated by Karlovitz number $\left(K a=\left(\alpha / S_{L} \eta_{k}\right)^{2}\right)$ of order unity [25]. $\alpha, S_{L}$, and $\eta_{k}$ are the thermal diffusivity, laminar flame speed and Kolmogorov length scale respectively. Because the Karlovitz number can be varied independently from the Reynolds number, it is important to note that flamelet combustion, and effects of the associated differential diffusion processes, can occur even at high Reynolds number, provided that $\mathrm{Ka}$ is small.

The physical basis for the localness requirement is the manner in which fluid mixes only with the composition field in its neighborhood (in physical space). Since the composition fields in reality are smooth, it is assumed that this neighborhood in physical space corresponds to a neighborhood in composition space.

The paper proceeds with a discussion of the applicability of PDF mixing models to turbulent flames with differential diffusion, leading to a method for accounting for differential diffusion in pairwiseexchange mixing models. This method is applied to the Interaction by Exchange with the Mean [26] and the Euclidean Minimum Spanning Tree [5] mixing models in order to account for differential diffusion. DNS data for turbulent premixed combustion with differential diffusion is then used to test whether the new mixing models improve the prediction of differential diffusion effects.

\section{PDF modelling of differential diffusion}

Numerous PDF mixing models have been presented in the literature on combustion modelling, however none satisfy the complete set of performance criteria presented by Subramaniam and Pope [5]. These models include Interaction by Exchange with the Mean (IEM) [26], modified Curl [27], mapping closure [28,29], Euclidean Minimum Spanning Tree (EMST) [5], multiple mapping conditioning [30], binomial [31], Fokker-Planck [32], and presumed scalar profile [33] approaches. Meyer [8] provides a further model, and a summary of various models' performance. Here, we consider these mixing models in light of the localness, length scale dependence, and differential diffusion criteria which are especially important in modelling of flamelet combustion, and note that: (1) the EMST model is the only multi-scalar model which enforces scalar-localness; (2) it is not clear that any of the mixing models referred to are able to account directly for the distribution of scalar-length scales occurring in flamelet combustion; and (3) none of the mixing models account for differential diffusion in a manner which enforces realizability (explained in Section 2.1 below).

In this paper we present a new method for accounting for differential diffusion in pairwise-exchange mixing models - such as the IEM, EMST and modified Curl models - which satisfies appropriate realizability conditions by applying a correction term. For the differential diffusion correction to satisfy conditions of realizability it is necessary for the mixing model to adjust the statistical weighting of the PDF particles, therefore this approach cannot be applied to transported PDF model formulations which require the statistical weighting of the PDF particles (or fields) to remain constant. Below, the differential diffusion correction is expressed in a general form that is applicable to any pairwise-exchange mixing model. Then, the practical application of this correction is presented for two pairwise-exchange mixing models: the EMST model is selected due to its ability to describe scalar localness; and modification of the simpler IEM model is also presented for purposes of comparison.

\subsection{Pairwise-exchange models with different mixing rates for each species}

A definition of pairwise-exchange mixing is given mathematically in Subramaniam and Pope [5], in which it is assumed that the statistical weightings of the PDF particles are not changed by the mixing model. Here we provide a more general definition 
which removes the assumption of constant particle weights. Pairwise-exchange mixing can be expressed as a matrix of interactions among all pairs in the ensemble of particles, with the interaction between the $p$ th and $q$ th particles satisfying:

$\left.\frac{d w^{(p)} Y_{\alpha}^{(p)}}{d t}\right|_{q}=-\left.\frac{d w^{(q)} Y_{\alpha}^{(q)}}{d t}\right|_{p}$

where $w^{(i)}$ is the particle weighting for the ith particle. The notation $d\left({ }^{(p)}\right) /\left.d t\right|_{(q)}$ is used to indicate the rate of change of quantity $(\cdot)$ associated with particle $p$ due to the interaction with particle $q$ only. Satisfaction of Eq. (3) guarantees that the weighted mean composition of the particle pair $p q$ is not changed by their interaction. The overall rate of change of $\left({ }^{(p)}\right)$ is obtained by summing the interactions of particle $p$ with all $N$ particles in the ensemble $(q \in\{1 \ldots N\})$, therefore pairwise conservation of mean quantities guarantees that the ensemble means are also conserved during mixing. The IEM and EMST mixing models have been formulated so that the particle weightings are not altered by the mixing process, such that the interaction of particle pair $p q$ takes the form

$\left.w^{(p)} \frac{d Y_{\alpha}^{(p)}}{d t}\right|_{q}=-a^{(p q)}\left(Y_{\alpha}^{(p)}-Y_{\alpha}^{(q)}\right)=-\left.w^{(q)} \frac{d Y_{\alpha}^{(q)}}{d t}\right|_{p}$,

with

$\left.\frac{d w^{(p)}}{d t}\right|_{q}=\left.\frac{d w^{(q)}}{d t}\right|_{p}=0$,

where the mixing coefficient $a^{(p q)}$ is specified by the mixing model. In the original IEM [26] and EMST [5] mixing models, the same value of $a^{(p q)}$ is used for every species being mixed between particles $p$ and $q$. A value of $a^{(p q)} \geqslant 0$ ensures: (1) that the difference $\left|Y_{\alpha}^{(p)}-Y_{\alpha}^{(q)}\right|$, which contributes to the variance, decreases or remains constant; and (2) that the composition remains within realizable bounds defined by the convex-hull of composition space.

\subsubsection{Conditions for realizability}

The conditions for realizability in a differentially diffusive mixture are presented by McDermott and Pope [10]. In the case of nonequal diffusivities, the diffusion process is not required to keep the composition within the convex-hull of composition space and McDermott and Pope provide two basic conditions for realizability: the sum of all $\mathrm{M}$ species mass fractions on each particle must remain equal to unity,

$\sum_{\alpha=1}^{M} Y_{\alpha}^{(i)}=1$

and individual mass fractions must remain bounded by zero

$0 \leqslant Y_{\alpha}^{(i)}$.

Satisfying Eqs. (6) and (7) also ensures that mass fractions of individual species never exceed unity.

Under certain conditions, noted in Section 1, it is appropriate for the mean scalar dissipation rates to depend on the diffusivity of individual species. Within the framework of a pairwise-exchange model, it is possible to achieve different scalar dissipation rates by specifying a different value of $a^{(p q)}$ for each species. A serious problem arises, however, since the pairwise-exchange no longer ensures that the mass fractions sum to unity (Eq. (6)). Note that the mixing models by Chen and Chang [7] and by Meyer [8] which otherwise account for differential diffusion quite successfully - both violate Eq. (6) when the mixing coefficients differ between species. A practical remedy which ensures that the sum of mass fractions remains equal to unity might be to solve the Chen and Chang or Meyer diffusion models for $M-1$ species and determine the remaining mass fraction from the condition that $\sum_{\alpha=1, M} Y_{\alpha}=1$, however this practice violates the conditions for boundedness in Eq. (7). Here, we propose a correction term that permits differential mixing, where each species has its own mixing coefficient $a_{\alpha}^{(p q)}$, and which satisfies the realizability conditions specified by Eqs. (6) and (7).

\subsubsection{A correction term to enforce realizability}

Where species dependent mixing factors, $a_{\alpha}^{(p q)}$, are employed, an additional term can be added to Eq. (4) to enforce realizability:

$\left.w^{(p)} \frac{d Y_{\alpha}^{(p)}}{d t}\right|_{q}=-a_{\alpha}^{(p q)}\left(Y_{\alpha}^{(p)}-Y_{\alpha}^{(q)}\right)+Y_{\alpha}^{(p)} \sum_{\beta=1}^{M} a_{\beta}^{(p q)}\left(Y_{\beta}^{(p)}-Y_{\beta}^{(q)}\right)$.

The additional term is analogous to the correction velocity employed in some models for the diffusion velocities in systems with differential diffusion (p. 16 in Ref. [34]). Summing Eq. (8) over $\alpha \in\{1 \ldots M\}$ gives $d\left\{\sum_{\alpha} Y_{\alpha}^{(p)}\right\} /\left.d t\right|_{q}=0$, satisfying Eq. (6). The individual mass fractions also remain bounded by zero since the second term on the right hand side in Eq. (8) tends to zero as $Y_{\alpha}^{(p)}$ approaches zero, and the first term on the right hand side is known to satisfy Eq. (7) provided that the mixing coefficients are all positive.

Conservation of means then requires an exchange of particle weight given by,

$\left.\frac{d w^{(p)}}{d t}\right|_{q}=-\sum_{\beta=1}^{M} a_{\beta}^{(p q)}\left(Y_{\beta}^{(p)}-Y_{\beta}^{(q)}\right)=-\left.\frac{d w^{(q)}}{d t}\right|_{p}$

According to Eq. (9) it is possible (although unlikely in practice) for some particle weights to decrease to zero. If the statistical weight of a particle decreases to zero it should be removed from the ensemble. Eqs. (8) and (9) satisfy the definition of a pairwise-exchange model, stated in Eq. (3), since,

$$
\begin{aligned}
\left.\frac{d w^{(p)} Y_{\alpha}^{(p)}}{d t}\right|_{q} & =w^{(p)} \frac{d Y_{\alpha}^{(p)}}{d t}+Y_{\alpha}^{(p)} \frac{d w^{(p)}}{d t}=-a_{\alpha}^{(p q)}\left(Y_{\alpha}^{(p)}-Y_{\alpha}^{(q)}\right) \\
& =-\left.\frac{d w^{(q)} Y_{\alpha}^{(q)}}{d t}\right|_{p}
\end{aligned}
$$

The new model does not guarantee that all species variances decay since the second term on the right hand side of Eq. (8) may have a magnitude greater than the first. In Appendix A we demonstrate that - according to the multi-component and mixture averaged transport models - differential diffusion does, in fact, permit generation of scalar variances (negative values of the normalized scalar mixing rate, $\left.\Lambda_{\alpha}=\widetilde{\chi}_{\alpha} / Y_{\alpha}^{\prime \prime 2}\right)$. Therefore we note that the requirement that mixing models should produce a decay of scalar variances in the case of mixtures containing species with equal diffusivities, as stated by Subramaniam and Pope [5], does not apply strictly to flows involving differential diffusion. Experimental observations of the production of conserved scalar variance in premixed flames have been reported by Barlow and coworkers [35].

If the joint-PDF of composition includes the specific enthalpy of the mixture, $h^{(p)}$, a correction term is required in order to achieve conservation of the mean specific enthalpy:

$\left.w^{(p)} \frac{d h^{(p)}}{d t}\right|_{q}=-a_{h}^{(p q)}\left(h^{(p)}-h^{(q)}\right)+h^{(p)} \sum_{\beta=1}^{M} a_{\beta}^{(p q)}\left(Y_{\beta}^{(p)}-Y_{\beta}^{(q)}\right)$.

Conservation of mass, momentum and kinetic energy demand that, if particle velocities are used in the PDF calculation and the particle weights change, a correction is added to the particle velocities. Particle velocities are not used in the zero-dimensional PDF calculations in this study, however the derivation of the velocity correction term is given in Appendix B. 


\subsubsection{Specification of differential mixing rates}

The set of species dependent mixing factors $a_{\alpha}^{(p q)}$ should satisfy the following criteria: First, in non-reacting high Reynolds number flow, for which it is assumed that the scalar gradient statistics are dependent only on the turbulent cascade, the mean scalar dissipation rates should not depend on diffusivity. Second, the rate at which species become decorrelated should have a dependence on diffusivity [21]. Satisfaction of both these criteria can be achieved by making $a_{\alpha}^{(p q)}$ a stochastic quantity (Private communication, Prof. S.B. Pope, Cornell University, 2009). The mean value of the mixing coefficient $E\left(a_{\alpha}^{(p q)}\right)$ controls the mean scalar dissipation rates of the species (which should be equal in the case of non-reacting high Reynolds number flow mixing), and the statistical distribution of $a_{\alpha}^{(p q)}$ should depend on the diffusivity of the respective species in order to control the rate of decorrelation between the species. In flows where the scalar gradients are altered by factors other than turbulent dissipation (e.g. by chemical reaction $[19,18]$, or by the initial or boundary conditions [20]) the scalar dissipation rates and $E\left(a_{\alpha}^{(p q)}\right)$ may depend on the species diffusivities.

Two modelling tasks present themselves: (1) to provide a model for the distribution of $a_{\alpha}^{(p q)}$ and (2) to provide a model for $E\left(a_{\alpha}^{(p q)}\right)$. We note that several PDF mixing models apply stochastic fluctuations to the mixing coefficient $[8,33,36]$, although it is kept the same for all species. For example Meyer and Jenny [33] apply a gamma-distribution model based on a turbulence frequency model by Jayesh and Pope [37]. These approaches may suggest a starting point for developing a model where the mixing coefficients' distributions depend on individual species' diffusivities. In the present study of premixed combustion, for simplicity, we adopt a deltafunction PDF (i.e. $a_{\alpha}^{(p q)}=E\left(a_{\alpha}^{(p q)}\right)$ ) and we turn our attention to the modelling of $E\left(a_{\alpha}^{(p q)}\right)$.

In the case of high Reynolds number mixing of a low Damköhler number mixture (where chemistry contributes little to the scalar gradients), $E\left(a_{\alpha}^{(p q)}\right)$ should be the same for each species and equal to the value of $a^{(p q)}$ in the standard pairwise-exchange models. For higher Damköhler numbers, where reaction contributes significantly to the scalar gradients, the scalar dissipation of reactive species depends both on the scalar diffusivity and the flame structure [19]. The value of $E\left(a_{\alpha}^{(p q)}\right)$ is model specific: in mixing models which do not account for localness, $E\left(a_{\alpha}^{(p q)}\right)$ should account for differences in the scalar diffusivity and scalar structure; and in mixing models which correctly account for scalar-localness, $E\left(a_{\alpha}^{(p q)}\right)$ should be adjusted to account only for differences in scalar diffusivities.

\subsection{The IEM-DD model for differential mixing rates}

\subsubsection{The standard IEM model}

The interaction by Exchange with the Mean model [26] for the evolution of the mass fraction $Y$ of species $\alpha$ on stochastic particle $p$ is given by,

$\frac{d Y_{\alpha}^{(p)}}{d t}=-\frac{1}{2} C_{Y} \omega\left(Y_{\alpha}^{(p)}-\left\langle Y_{\alpha}\right\rangle\right)$

where $\left\langle Y_{\alpha}\right\rangle$ is the weighted mean given by a number of particles, $N$, with weights $w^{(i)}$,

$\left\langle Y_{\alpha}\right\rangle=\frac{\sum_{i=1}^{N} w^{(i)} Y_{\alpha}^{(i)}}{\sum_{i=1}^{N} w^{(i)}}$.

The IEM model is a pairwise-exchange model since Eq. (13) can be used to express Eq. (12) as a sum of $N$ pairwise interactions involving particle $p$. Scalar values of individual particles are subject to a deterministic relaxation towards the local mean value. The IEM mixing process does not change the shape of the PDF. The model parameter $C_{Y}$ is the ratio between the mechanical and scalar mixing rates and it is usually given the value of 2.0 for high Reynolds number mixing of a passive scalar [38].

\subsubsection{The IEM-DD model}

We employ a set of coefficients $C_{\beta}$ in the IEM-DD model which, in order to account for differential mixing rates, can differ among species. Applying the approach developed in Section 2.1, the mass fractions evolve according to,

$\frac{d Y_{\alpha}^{(p)}}{d t}=-\frac{C_{\alpha} \omega}{2}\left(Y_{\alpha}^{(p)}-\left\langle Y_{\alpha}\right\rangle\right)-Y_{\alpha}^{(p)} \sum_{\beta=1}^{M}-\frac{C_{\beta} \omega}{2}\left(Y_{\beta}^{(p)}-\left\langle Y_{\beta}\right\rangle\right)$.

The particle weighting must also change in order to conserve the weighted species averages,

$$
\frac{d w^{(p)}}{d t}=w^{(p)} \sum_{\beta=1}^{M}-\frac{C_{\beta} \omega}{2}\left(Y_{\beta}^{(p)}-\left\langle Y_{\beta}\right\rangle\right) .
$$

The scalar variance equation corresponding to the IEM-DD model is derived in Appendix C. It is notable that, due to the last term in Eq. (14), it is possible for the IEM-DD model to generate scalar variance.

The IEM-DD mixing coefficients $C_{\beta}$ determine the species mixing rates and they should account for flame structure and differential diffusion. Richardson et al. [19] provide a method for modelling the mixing rates of individual species within a turbulent flame, based on strained laminar flame solutions. In the simulations described below, however, the individual species mixing rates are taken directly from the DNS in order to avoid uncertainty due to their modelling. Eqs. (14) and (15) are advanced with first-order Euler integration, and the values of the mixing coefficients $C_{\beta}$ were adjusted iteratively to match, within $1 \%$, the mixing rates measured in the DNS.

\subsection{The EMST-DD model for differential diffusion}

\subsubsection{The standard EMST model}

The EMST mixing model is based on interactions between particles that are neighboring in composition space. It provides an extension of the mapping closure for multiple scalars. A complete description of the EMST and further references are provided in [5].

At any given time a subset of $N_{T}$ particles is chosen for mixing from the ensemble of $\mathrm{N}$ particles in one grid cell, based on an age property associated with each particle. The age property causes different particles to be excluded from the subset over time and reduces stranding in composition space [5]. A Euclidean minimum spanning tree is constructed on this subset of $N_{T}$ particles so that each particle is connected with at least one neighbor particle. Pairwise mixing occurs along every branch of the tree. The contribution of the EMST model to the change of $Y_{\alpha}^{(p)}$ is given by,

$w^{(p)} \frac{d Y_{\alpha}^{(p)}}{d t}=\sum_{q=1}^{N}-a B_{p q}\left(Y^{(p)}-Y^{(q)}\right)$.

' $a$ ' is a global mixing coefficient, and $B_{p q}$ is a mixing coefficient that depends on the location of the branch $(p q)$ within the tree. The specification of model coefficients $B_{p q}$ and $a$, and the resulting variance decay rate is described in Subramaniam and Pope [5]. Note that in Eq. (41) or Ref. [5] Subramaniam and Pope define $\boldsymbol{B}$ as a vector containing the mixing coefficient for every edge on the minimum spanning tree, whereas in Eq. (16) $\boldsymbol{B}$ is an $N \times N$ matrix with values corresponding to the vector in [5] and containing zeros where particles $p$ and $q$ are not neighbors in the minimum spanning tree. The implementation of the EMST model and standard model coefficients provided by Ren et al. [39] has been used in this work. 
' $a$ ' is a global mixing coefficient, and $B_{p q}$ is a mixing coefficient that depends on the location of the branch $(p q)$ within the tree (it is zero if particles $p$ and $q$ are not neighbors in the tree, or if either particle is not included in the set of $N_{T}$ active particles). The specification of model coefficients $B_{p q}$ and $a$, and the resulting variance decay rate is described in Subramaniam and Pope [5]. The implementation of the EMST model and standard model coefficients provided by Ren et al. [39] has been used in this work.

\subsubsection{The EMST-DD model}

We derive the EMST-DD model for differential diffusion by applying the methodology developed in Section 2.1 (Eqs. (8) and (9)) to the standard EMST model. The composition of particle $p$ then evolves according to

$w^{(p)} \frac{d Y_{\alpha}^{(p)}}{d t}=\sum_{q=1}^{N}\left\{-a C_{\alpha} B_{p q}\left(Y_{\alpha}^{(p)}-Y_{\alpha}^{(q)}\right)+Y_{\alpha}^{(p)} \sum_{\beta=1}^{M} a C_{\beta} B_{p q}\left(Y_{\beta}^{(p)}-Y_{\beta}^{(q)}\right)\right\}$,

with the rate of change of the particle weight given by,

$\frac{d w_{\alpha}^{(p)}}{d t}=-\sum_{q=1}^{N} \sum_{\beta=1}^{M} a C_{\beta} B_{p q}\left(Y_{\beta}^{(p)}-Y_{\beta}^{(q)}\right)$.

A model for the functional form of the species-dependent mixing coefficients remains to be specified. We propose the following model:

$C_{\alpha}=\frac{E\left(a_{\alpha}^{(p q)}\right)}{a^{(p q)}}=1+C_{K}\left(\frac{1}{L e_{\alpha}}-1\right)$,

in which the species dependent mixing coefficients depend on the species Lewis numbers $L e_{\alpha}$, and a model parameter $C_{K}$ which takes a value between zero and unity. When $C_{K}$ equals zero the standard EMST model is recovered. When $C_{K}$ equals unity, the rate at which mass is transferred between two particles is proportional to the diffusivity and the scalar-difference between the pair, and 'differential mixing' occurs. Based on the discussion in the introduction, we expect differential diffusion to become important in the flamelet regime of combustion, and to be unimportant in high-Reynolds number mixing of inert or low-Damköhler number mixtures. This suggests that $C_{K}$ should be a function of the combustion regime, for example, measured by the Karlovitz number: i.e. $C_{K} \rightarrow 0$ for $\mathrm{Ka} \gg 1$, and $C_{K} \rightarrow 1$ for $\mathrm{Ka} \rightarrow 0$. Non-zero values of $C_{K}$ may also be used to model the effects of differential diffusion on scalar dissipation rates in regions of flow where scalar gradients are strongly influenced by the boundary or initial conditions, for example in the DNS of Juneja and Pope [20]. In this investigation we report results for the $C_{K}=0$ (which is the standard EMST model) and for the extreme case of $C_{K}=1$.

\subsubsection{Implementation of the EMST-DD model}

The differential diffusion correction in Eq. (17) is implemented as a 'wrapper' around the implementation of the EMST model by Ren et al. [39]. Integrating the standard EMST model over the time increment $\Delta t$ changes the composition of particle $p$ by $\Delta Y_{\alpha E M S T}^{(p)}$. The EMST-DD correction is applied at each time step according to:

$\Delta Y_{\alpha E M S T-D D}^{(p)}=a_{D D}\left[C_{\alpha} \Delta Y_{\alpha E M S T}^{(p)}-Y_{\alpha}^{(p)} \sum_{\beta=1}^{M} C_{\beta} \Delta Y_{\beta E M S T}^{(p)}\right]$.

The parameter $a_{D D}$ is adjusted iteratively so that Eq. (20) produces the required the variance function dissipation rate. In the simulations presented below, the variance function dissipation rate used in the EMST-DD calculations has been extracted from the DNS data.

\section{Simulation data and analysis}

\subsection{Turbulent flame configuration}

The new PDF mixing models are tested by comparison with DNS data. The DNS configuration has been devised in order to isolate the effects of the micro-mixing models from effects of chemical and spatial transport models in the PDF method. The configuration involves premixed combustion of a methane-air mixture in twodimensional decaying turbulence in an $8 \mathrm{~mm}$ square domain with periodic boundaries. A circular, laminar flame kernel is positioned at the center of the domain at the start of the simulation. This method of initialization gives a scalar field with length scales imposed entirely by flame propagation processes, and with no correlation with the turbulence field. Two calculations are performed starting from the same initial conditions: first, a non-reacting simulation with all chemical reaction rates set to zero; and second, with the chemical reactions activated.

While two-dimensional turbulent mixing is qualitatively different from three-dimensional turbulence, it does permit useful investigation of micro-mixing model properties [40]. To provide further support to the conclusions of this study, we show that mixing statistics from the 2D DNS display similar trends to mixing statistics from a 3D-turbulent Bunsen flame (Fig. 3) [19,41] at very similar thermo-chemical conditions. One-dimensional PDF simulation results for the 3D Bunsen flame, which are provided as supplementary material, also support the conclusions based on the 2D DNS data.

The details of the 2D DNS configuration are summarized in Table 1. The initial scalar field is obtained from a calculation of a circular laminar flame centered in the DNS domain (the initial pressure, unburned temperature, and equivalence ratio for the laminar calculation are $1 \mathrm{~atm}, 800 \mathrm{~K}$, and $\phi=0.7$ respectively). The laminar flame calculation results in an approximately circular region of products at the center of the DNS domain (shown in Fig. 1a) with diameter $4 \mathrm{~mm}$ and $1.21 \mathrm{~atm}$ pressure.

At the start of the turbulent combustion simulation, $t=0$, a turbulent velocity field is added to the velocity field of the initial $2 \mathrm{D}$ laminar flame solution. The turbulent velocity field was initialized using an isotropic, homogeneous turbulent kinetic energy spectrum given by [42]

$E(k)=\frac{32}{3} \sqrt{\frac{2}{\pi}} \frac{u^{\prime 2}}{k_{e}}\left(\frac{k}{k_{e}}\right)^{4} \exp \left[-2\left(\frac{k}{k_{e}}\right)^{2}\right]$.

Here $k_{e}$ is the most energetic wave number, given by

Table 1

Initial turbulence and combustion parameters for the chemically reacting 2D premixed flame configuration. The Values are based on the unburnt mixture where $\alpha=1.2 \times 10^{-4} \mathrm{~m}^{2} \mathrm{~s}^{-1}$ and $v=8.2 \times 10^{-5} \mathrm{~m}^{2} \mathrm{~s}^{-1}$, and the laminar fame speed $S_{L}$ and thickness $\delta_{f}$ are $1.7 \mathrm{~ms}^{-1}$ and $0.3 \mathrm{~mm}$ respectively.

\begin{tabular}{ll}
\hline Variable & Value \\
\hline$u^{\prime}\left(\mathrm{m} \mathrm{s}^{-1}\right)$ & 6.8 \\
$L_{T}(\mathrm{~mm})$ & 2.0 \\
$L_{11}(\mathrm{~mm})$ & 0.23 \\
$\eta_{k}=\left(v^{3} / \epsilon\right)^{1 / 4}(\mathrm{~mm})$ & 0.043 \\
$\tau_{t}=L_{T} / u^{\prime}(\mathrm{ms})$ & 0.29 \\
$\tau_{f}=\delta_{f} / S_{L}(\mathrm{~ms})$ & 0.18 \\
$u^{\prime} / S_{L}$ & 4.0 \\
$L_{T} / \delta_{f}$ & 6.7 \\
$\mathrm{Da}=\tau_{t} / \tau_{f}$ & 1.6 \\
$\mathrm{Ka}=\left(\alpha / \mathrm{S}_{L} \eta_{k}\right)^{2}$ & 2.7 \\
\hline
\end{tabular}




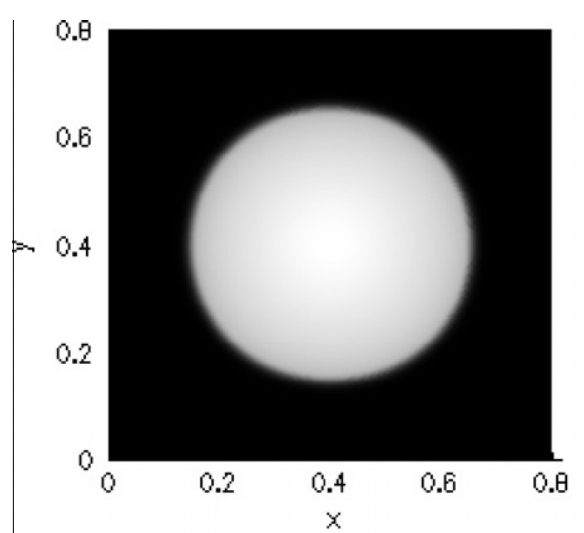

(a)

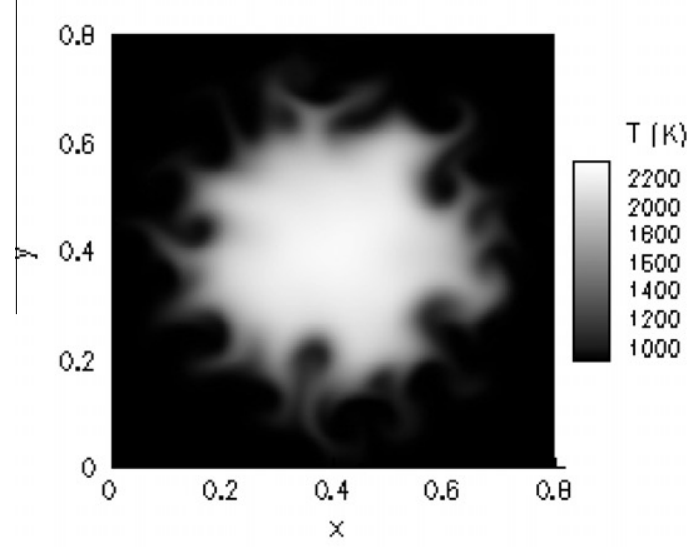

(b)

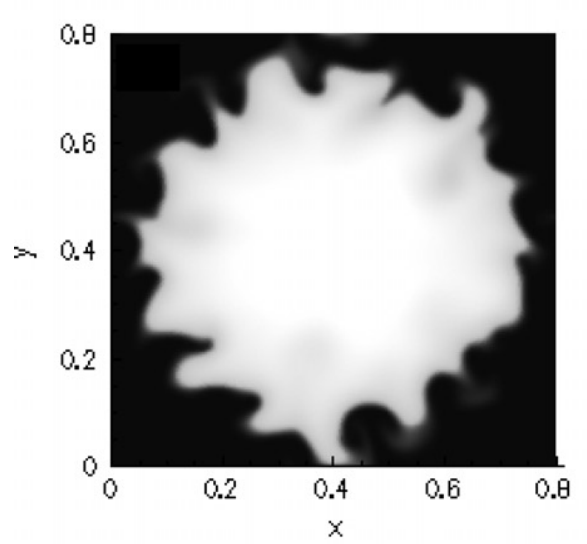

(c)

Fig. 1. Two-dimensional premixed flame DNS configuration, showing the instantaneous temperature field for (a) $t=0$; (b) inert case at $t=\tau_{f}$; and (c) chemically reacting case at $t=\tau_{f}$. Axis units are centimeters.

$k_{e}=\sqrt{\frac{4 \epsilon}{10 v u^{\prime 2}}}$.

The turbulence parameters were set according to Table 1 by choosing $\epsilon$ such that the desired autocorrelation integral scale $\left(L_{11}\right)$ was obtained:

$L_{11}=\frac{8}{3 k_{e}} \sqrt{\frac{2}{\pi}}$
The Reynolds number based on the integral length scale $L_{11}$ is 19. The low Reynolds number indicates that the DNS does not contain a wide range of turbulent scales. This study assesses the impact of flame structures on the modelling of small-scale mixing effects, meaning that the larger-scale dynamics are less critical, provided that the large-scales do impose a realistic unsteady variation of flame strain and curvature. Section 4.1 shows that the 2D DNS does reproduces important mixing effects seen in 3D turbulent DNS [19] and it is therefore useful for testing the ability of mixing models to describe the physical processes associated with differential diffusion.

PDF mixing models have been evaluated by comparing conditional and unconditional moments between the PDF method predictions and the DNS results. The statistical convergence of the DNS data has been evaluated by computing confidence intervals for the estimates of the first and second moments of the progress variable and its reaction source term. The confidence intervals were computed by dividing the DNS domain into four equal squares and treating each quarter as an independent sample (in fact the samples are not strictly independent). The $95 \%$ confidence intervals for the mean and root-mean-square at $t=\tau_{f}$ were less than $2.5 \%$ and $5 \%$ of the absolute values for the progress variable and its reaction rate respectively. This indicates that the DNS data provides sufficient statistical convergence to verify the conclusions of this study.

The same initial condition is used both for the non-reacting and reacting simulations. The temperature fields of the inert and chemically reacting simulations are shown in Fig. 1b and c after one flame timescale $\left(\tau_{f}=0.18 \mathrm{~ms}\right.$, based on the $S_{L}$ and flame thickness $\left(\delta_{f}\right)$ values with Table 1$)$.

\subsection{DNS methods}

The simulations were performed using the DNS code S3D, which solves the fully compressible Navier-Stokes, species and energy equations with a fourth-order Runge-Kutta method for time integration and eighth-order explicit spatial differencing $[43,44]$. A uniform $20 \mu \mathrm{m}$ grid spacing was employed throughout (i.e. $400 \times 400$ grid points). The simulations were advanced with 2 ns time steps.

Chemical reaction was modelled using a reduced mechanism with low temporal stiffness developed from the detailed GRI-1.2 scheme [45]. Details of the reduction methodology and validation of the reduced mechanism can be found in Ref. [46]. CHEMKIN and TRANSPORT software libraries $[47,48]$ were linked with S3D to evaluate reaction rates, thermodynamic, and mixture-averaged transport-properties. Note that the 3-D Bunsen flame calculation [19] which is discussed below, employed the same thermochemistry but different, constant Lewis-number, transport models. The Lewis numbers are given in Table 2 .

\subsection{PDF methods}

The two-dimensional DNS configuration has been modelled by following the zero-dimensional constant-volume PDF formulation

Table 2

Species Lewis numbers.

\begin{tabular}{llll}
\hline Species & Le & Species & Le \\
\hline $\mathrm{H}_{2}$ & 0.29 & $\mathrm{CH}_{3}$ & 0.97 \\
$\mathrm{H}$ & 0.17 & $\mathrm{CH}_{4}$ & 0.96 \\
$\mathrm{O}$ & 0.69 & $\mathrm{CO}$ & 1.07 \\
$\mathrm{O}_{2}$ & 1.08 & $\mathrm{CO}_{2}$ & 1.34 \\
$\mathrm{OH}$ & 0.70 & $\mathrm{CH}_{2} \mathrm{O}$ & 1.25 \\
$\mathrm{H}_{2} \mathrm{O}$ & $\mathrm{N}_{2}$ & 1.04 \\
$\mathrm{HO}_{2}$ & 0.82 & & \\
\hline
\end{tabular}


described by Bisetti et al. [40]. Spatial transport is neglected because the DNS domain is periodic and, considering scales greater than the size of the DNS domain, mean scalar gradients are zero. The thermo-chemical modelling is identical to that in the DNS. A zero-order splitting method is applied with 2 ns time steps. The chemical reaction step is integrated explicitly with a second-order Runge-Kutta scheme [49] using the same 2 ns time step as the DNS. Molecular mixing has been modelled using the standard IEM and EMST models, and using the differential diffusion versions of the IEM and EMST differential diffusion models introduced above. In all cases, mixing is performed on a state vector comprising species mass fractions and the specific enthalpy. The IEM-DD and EMST-DD models also adjust the statistical weighting of the individual particles. The mixing rates, provided as time-dependent input parameters to all of the mixing models, are determined from the DNS data. We provide the IEM, EMST, and EMST-DD with a single mixing rate, equal to $\Lambda_{c}$. Where $c$ is the progress variable, which we define in relation to the burned and unburned $\mathrm{O}_{2}$ mass fraction as $c=\left(Y_{O_{2}}-Y_{O_{2}}^{u}\right) /\left(Y_{O_{2}}^{b}-Y_{O_{2}}^{u}\right)$. In the IEM simulation, the rate of mixing is $\Lambda_{c}$ for all species, while for the EMST and EMST-DD models the mixing coefficients are controlled so that the mixing rate of the variance function (defined in Ref. [5]) equals $\Lambda_{c}$. The EMST-DD model is provided with the individual species' Lewis numbers given in Table 2. The IEM-DD model is provided with the DNS value of $\boldsymbol{\Lambda}$ for each variable in the state vector.

The PDF simulations used 500 particles, which is sufficient to recover unconditional moments within tight tolerances. To increase the sample size, for the evaluation of conditional statistics, each PDF simulation was repeated 20 times. The relative computation times for evaluation of the IEM, IEM-DD, EMST, and EMST-DD mixing models were $1.0,2.3,76$, and 78 units of processor time respectively. For the configuration simulated here, the adjustment of the IEM-DD and EMST-DD mixing rates converges rapidly, usually on the first iteration for each time step. The additional processor effort associated with the IEM-DD and EMST-DD models, compared to the standard IEM and EMST models respectively, is negligible compared with the time for chemistry integration.

\section{Results and discussion}

\subsection{Mixing characteristics of turbulent premixed combustion}

The DNS of two-dimensional turbulence leads to a highly wrinkled temperature field in both the inert and chemically reacting cases, seen at $t=\tau_{f}$ in Fig. $1 \mathrm{~b}$ and $\mathrm{c}$. The 2D DNS data are reported at $t=\tau_{f}$ so that the flame structure has had time to respond to the flow field. Based on the reference laminar flame

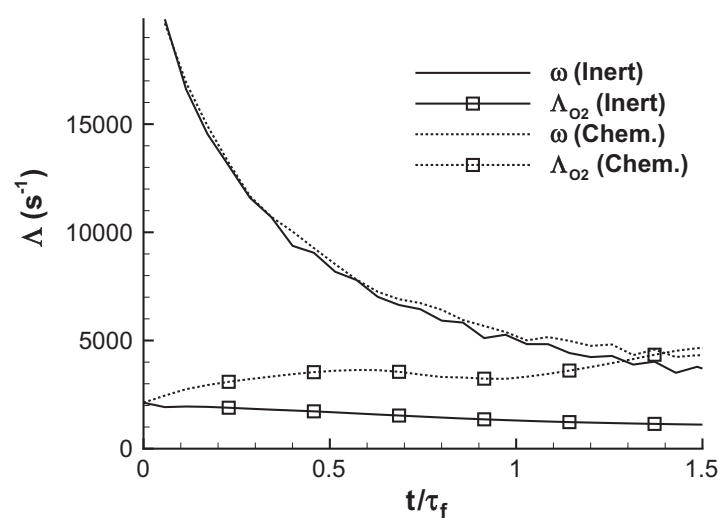

Fig. 2. The temporal evolution of the turbulent frequency $\omega$ and the $\mathrm{O}_{2}$ mixing rate $\Lambda_{\mathrm{O} 2}$ for the inert and chemically reacting 2D DNS cases.
Table 3

Turbulent combustion parameters for the Bunsen flame $\left(S_{L}=1.7 \mathrm{~ms}^{-1}, \delta_{f}=0.3 \mathrm{~mm}\right)$.

\begin{tabular}{llllll}
\hline Location & $\mathrm{Ka}$ & $\mathrm{Da}$ & $u^{\prime} / \mathrm{S}_{L}$ & $l_{t} / \delta_{l}$ & $\mathrm{Re}_{t}$ \\
\hline$x / L_{x}=0.25$ & 7.8 & 0.31 & 5.6 & 1.7 & 61 \\
$x / L_{x}=0.5$ & 7.2 & 0.34 & 5.4 & 1.7 & 59 \\
$x / L_{x}=0.75$ & 4.0 & 0.58 & 4.0 & 2.3 & 58 \\
\hline
\end{tabular}

quantities and the parameters of the initial turbulence field in Table 1, the chemically reacting case has an initial Damköhler number $\mathrm{Da}=1.6$, and Karlovitz number $\mathrm{Ka}=2.7$, suggesting that combustion takes place in the the thin reaction zones regime [25]. The value of Da increases as the turbulence decays, tending to make the combustion more flamelet-like. In contrast, the inert case represents the extreme case of passive-scalar mixing ( $\mathrm{Da}=0$ ).

The turbulent frequency and the progress variable mixing rate $\left(\Lambda_{c}\right)$ evaluated in the DNS are shown in Fig. 2. Chemical reaction causes little difference between the turbulent frequency in the inert and reacting cases. But after the initial condition, where $\Lambda_{c}$ results from the initial laminar flame solution, the mixing of progress variable does differ in the two cases: in the inert case, the laminar flame profile is disrupted by the turbulent flow and diffuses such that $\Lambda_{c}$ decays throughout the simulation; in the chemically reacting case, flame propagation maintains the steep gradients of the flame fronts while they are distorted by the turbulence. The mixing rate $\Lambda_{c}$ in the reactive case consequently increases relative to the inert case, exceeding the turbulent frequency at $1.3 \tau_{f}$.

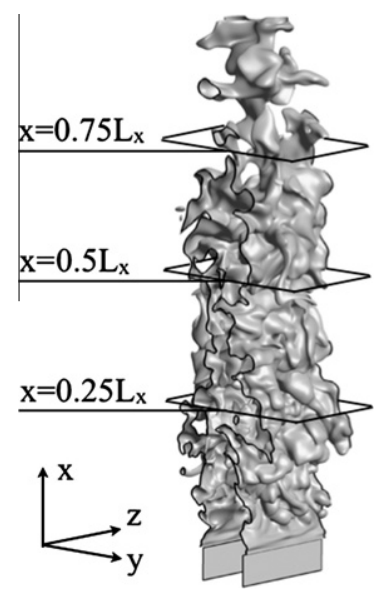

Fig. 3. Premixed Bunsen flame DNS configuration, showing the instantaneous $c=0.65$ iso-surface and measurement locations at $x / L_{x}=0.25,0.5$, and 0.75 .

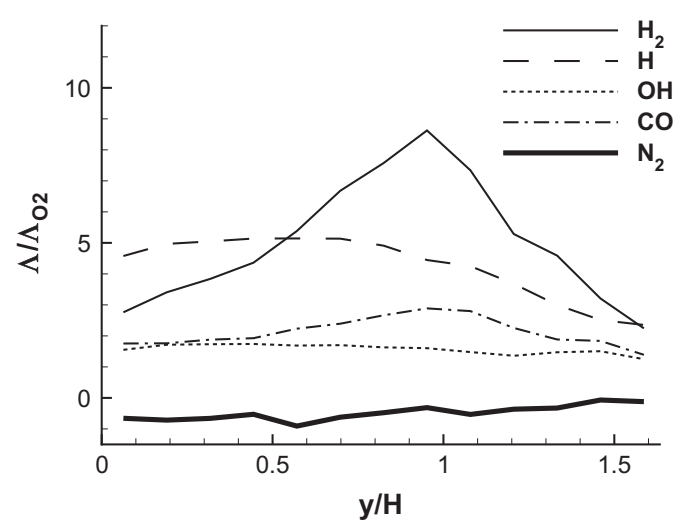

Fig. 4. Ratios for $\Lambda$ between selected species $\left(\mathrm{H}_{2}, \mathrm{H}, \mathrm{OH}, \mathrm{CO}, \mathrm{N}_{2}\right)$ and $\mathrm{O}_{2}$, in the Bunsen DNS at $x / L_{x}=0.5$. 
The mixing and combustion regimes displayed in the inert and chemically reacting 2D DNS bracket the range of combustion conditions found in the 3D turbulent Bunsen flame simulation [19]. The Karlovitz and Damköhler numbers for the 3D Bunsen case are given in Table 3, indicating that the Bunsen flame is also characterized by the thin reaction zones regime. Note that in the $3 \mathrm{D}$ Bunsen flame [19] the center-line turbulence frequency and progress variable mixing rate both decayed from approximately $20,000 \mathrm{~s}^{-1}$ at $x / L_{x}=0.25-5000 \mathrm{~s}^{-1}$ at $x / L_{x}=0.75$, where $L_{x}$ is the stream-wise length of the DNS domain shown in Fig. 3. This variation of turbulence frequencies is similar to the variation in the $2 \mathrm{D}$ DNS calculations, supporting the case for qualitative comparison between the 2D and 3D Bunsen data sets.

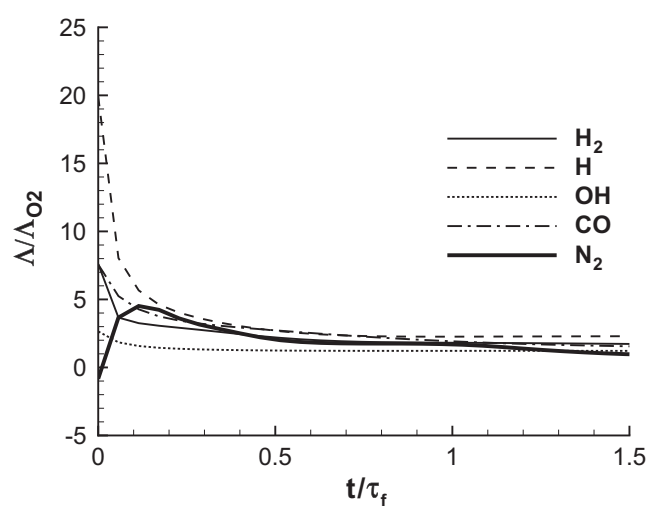

(a)

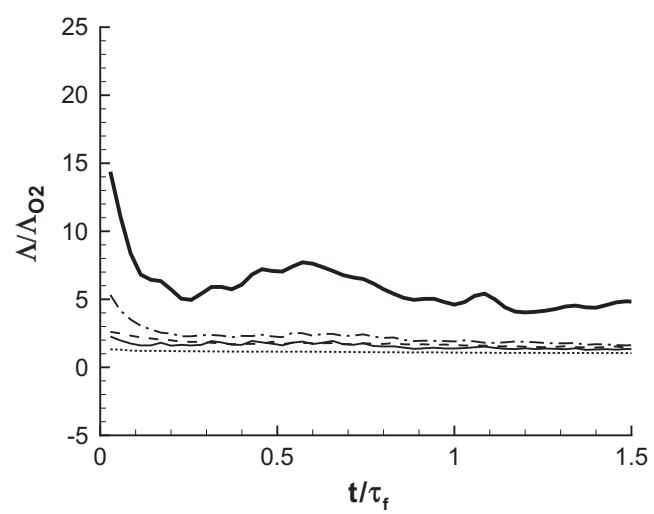

(c)

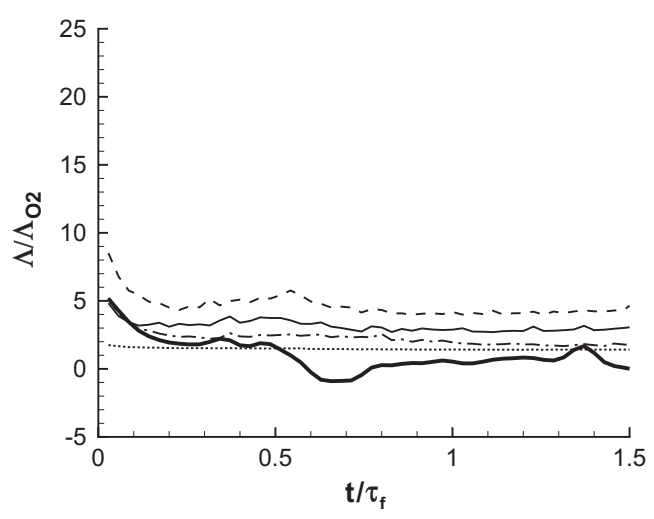

(e)
The variation of the species mixing rates $(\Lambda)$ of $\mathrm{H}_{2}, \mathrm{H}, \mathrm{OH}, \mathrm{CO}$, and $\mathrm{N}_{2}$ is presented for the axial location $x / L_{x}=0.5$ of the 3D Bunsen flame in Fig. 4, and for the inert and chemically reacting 2D DNS data in Fig. 5a and b respectively. The data show that intermediate species in the flame (such as atomic hydrogen) mix up to twenty times faster than major species, such as $\mathrm{O}_{2}$. Both the $2 \mathrm{D}$ and $3 \mathrm{D}$ data show that the mixing rate ratios can greatly exceed the inverse of the Lewis number ratios $\left(\mathrm{Le}_{\mathrm{O} 2} / \mathrm{Le}_{H} \approx 6\right)$, which implies that the mixing rate ratios can not be attributed solely to differences in diffusivities. The analysis of the 3D Bunsen data in Ref. [19] indicates that the elevated mixing rates are due to gradients associated with flame structures, and it was seen that the ability of flame propagation to enhance the mixing rates of intermediate

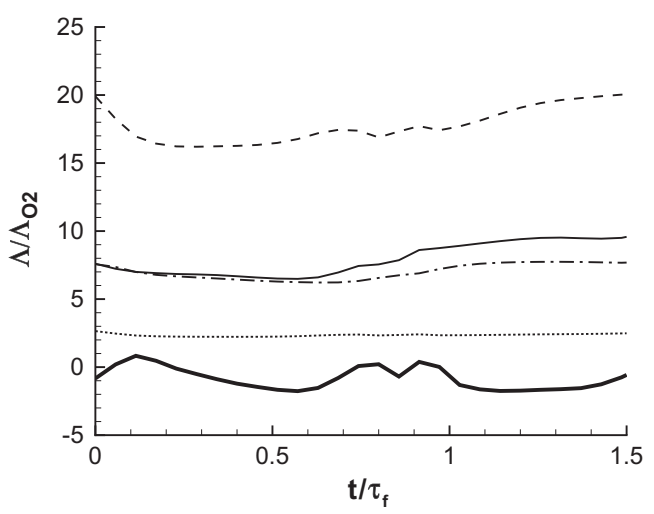

(b)

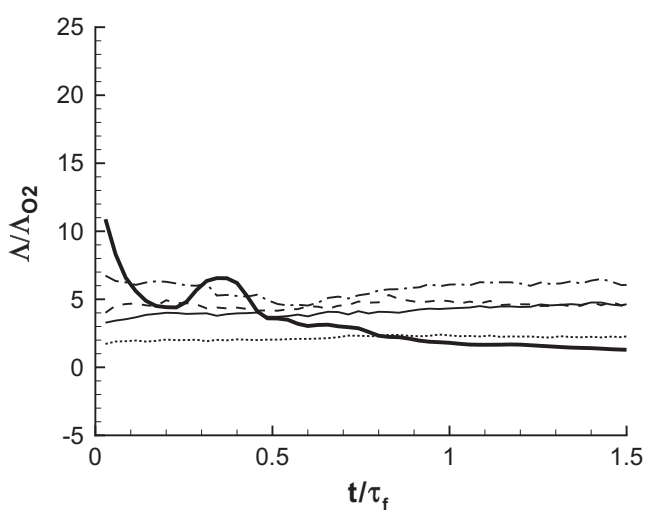

(d)

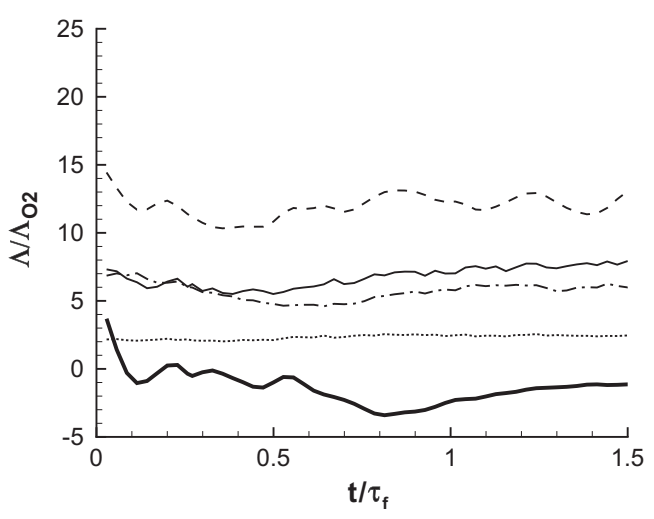

(f)

Fig. 5. Temporal evolution of the $\Lambda$ ratios between selected species $\left(\mathrm{H}_{2}, \mathrm{H}, \mathrm{OH}, \mathrm{CO}, \mathrm{N}_{2}\right)$ and $\mathrm{O}_{2}$ in the inert (left column) and chemically reactive (right column) $2 \mathrm{D}$ DNS. The top row is the DNS data, middle row is the EMST predictions, and the bottom row is the EMST-DD predictions. 
species increases with Damköhler number. This conclusion is supported by the inert 2D data where, in the absence of flame propagation, the mixing rate ratios rapidly decay from their initial laminar flame values towards unity. In the chemically reacting 2D simulation, however, the mixing rate ratios remain close to their initial values.

While it may be of limited practical significance in premixed methane combustion, it is also instructive to note that $\Lambda_{N_{2}}$ can be negative. As was discussed in Section 2.1, this phenomenon occurs in the premixed flame because, since $\mathrm{N}_{2}$ does not participate in any chemical reactions in this DNS, the variance of $N_{2}$ is entirely due to differential diffusion. See Appendix A for further explanation of the variance source term provided by differential diffusion.

The variation among mixing rates, $\Lambda_{\alpha}$, highlights the importance of flame structure, or localness in composition space, and differential diffusion for accurate prediction of the mixing in turbulent combustion. As such, prediction of the variance decay rates observed in the DNS provides a relevant performance criterion for the application of mixing models to real turbulent flames.

\subsubsection{Mixing statistics}

The accuracy of PDF mixing models can be evaluated by comparing the statistics of the diffusion rate $\left(\Gamma_{\alpha}\right)$ computed in the DNS and PDF calculations. Conditioning the diffusion rates on progress variable (whose sample-space variable is denoted $\zeta$ ) permits an assessment of how successfully mixing models predict the diffusion associated with the flame structures. The Favre conditional

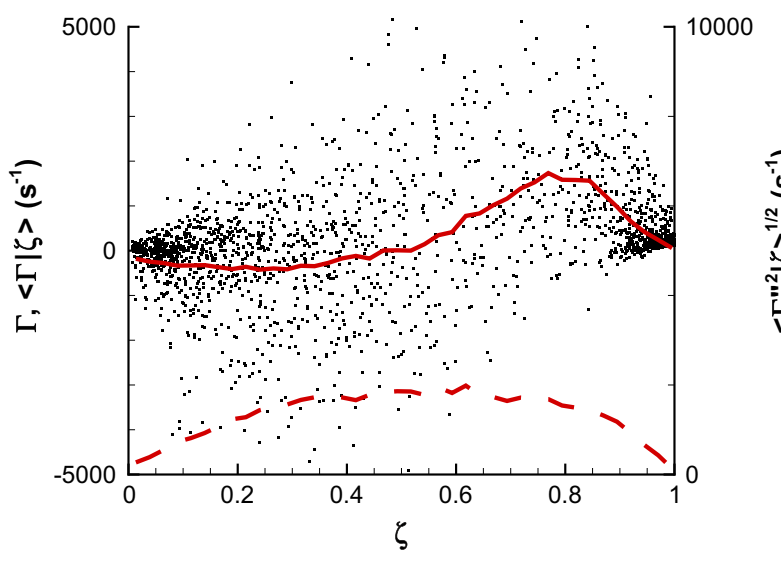

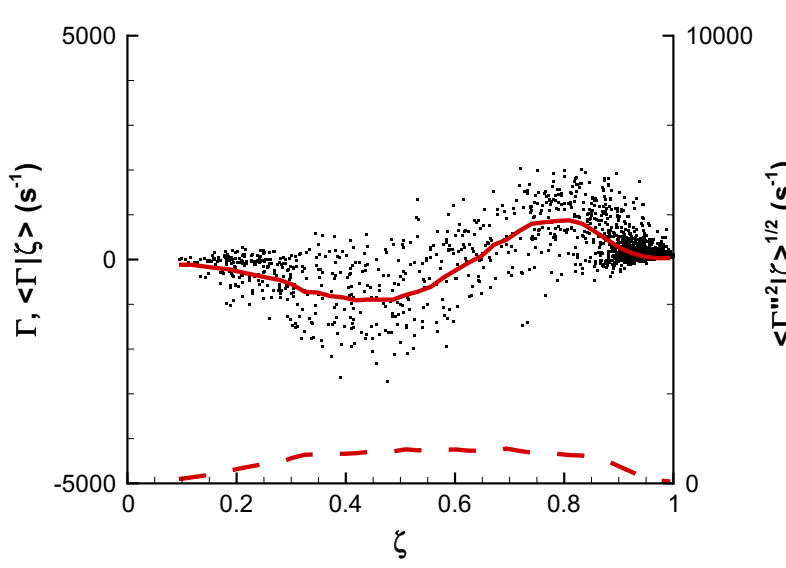

(b) $x / L x=0.75$

Fig. 6. $\mathrm{O}_{2}$ diffusion rates across progress variable space in the Bunsen flame DNS at $x / L_{x}=0.25$ and 0.75 . The conditional mean (solid) and rms (dashed) are also shown. average is written $\langle\Gamma \mid \zeta\rangle$, and the conditional root mean square (rms) value is written $\left\langle\Gamma^{\prime \prime 2} \mid \zeta\right\rangle^{1 / 2}$. Conditional statistics for the diffusion rate of $\mathrm{O}_{2}$ mass fraction (which is linearly related to the progress variable diffusion rate) are presented for the 3D Bunsen flame and the 2D DNS in Figs. 6 and 7 respectively. Figure 6 contains data from all cross-stream positions in order to ensure that all progress variable space is populated. The maximum conditionally averaged diffusion rate of $\mathrm{O}_{2}$ is greater approaching the Bunsen jet inlet, where the mean strain (reported in Ref. [46]) is higher. The ratio of the conditional rms to the conditional mean diffusion rates is higher at low Damköhler numbers (i.e. closer to the Bunsen jet inlet, or in the inert 2D DNS), because the influence of the flame structure on mixing becomes less important relative to the influence of turbulent mixing.

Figures 8 and 9 present conditional means and the rms values of $\mathrm{H}_{2}$, CO, and $\mathrm{N}_{2}$ species mass fractions for the Bunsen and 2D DNS cases respectively. The Bunsen data shows the progression of the composition from the flamelet profile imposed at the inlet, through the highly strained flow in the first quarter of the domain, and subsequent recovery of flamelet-like profiles as the turbulence decays downstream. The intense turbulence in the near field of the jet causes a reduction in the conditionally averaged CO mass fraction,

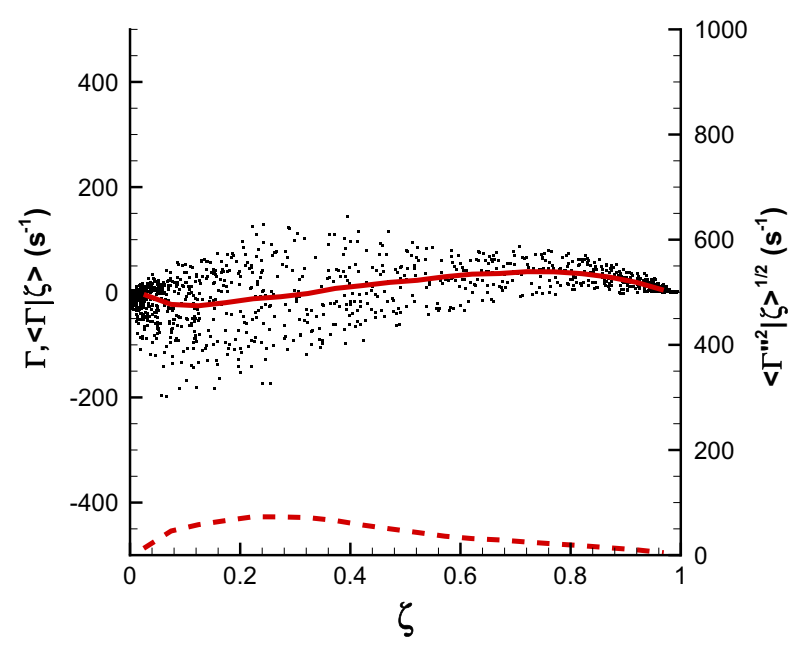

(a)

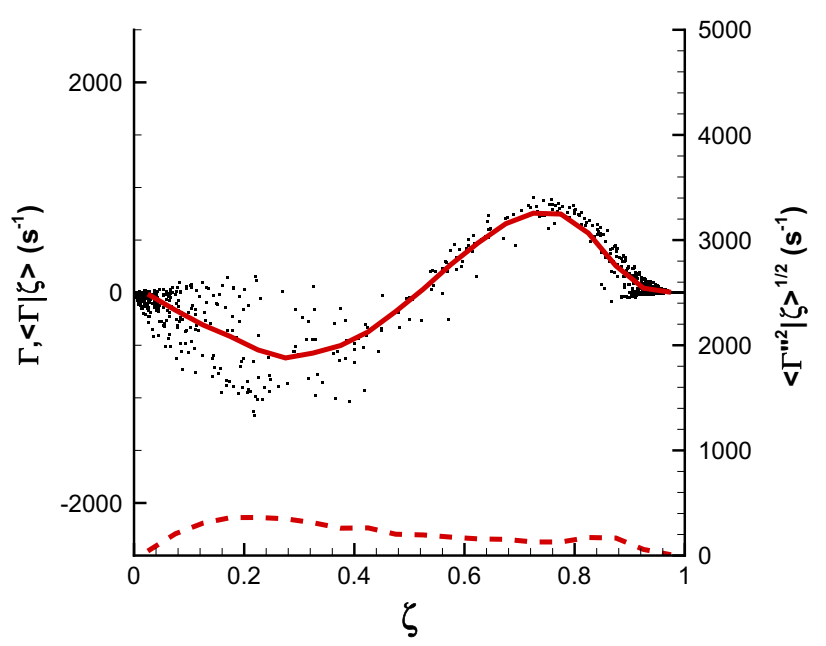

(b)

Fig. 7. $\mathrm{O}_{2}$ diffusion rates across progress variable space in the inert (top) and chemically reacting (bottom) 2D DNS at $t=\tau_{f}$. The conditional mean (solid) and rms (dashed) are also shown. 
reaching a minimum close to $x / L_{x}=0.25$. The composition profiles subsequently approach the original laminar flamelet profile as the strain rates and flame curvatures decrease downstream. The conditional scatter of the $\mathrm{CO}$ and $\mathrm{H}_{2}$ mass fractions shown in Fig. 8 are also seen to decay downstream.

The presence of differential diffusion is seen in the $\mathrm{H}_{2}$ profile in Fig. 8a. Differential diffusion leads to curvature in the conditional $\mathrm{H}_{2}$ mass fraction profiles in the non-reactive mixture $(\zeta<0.6)$. This

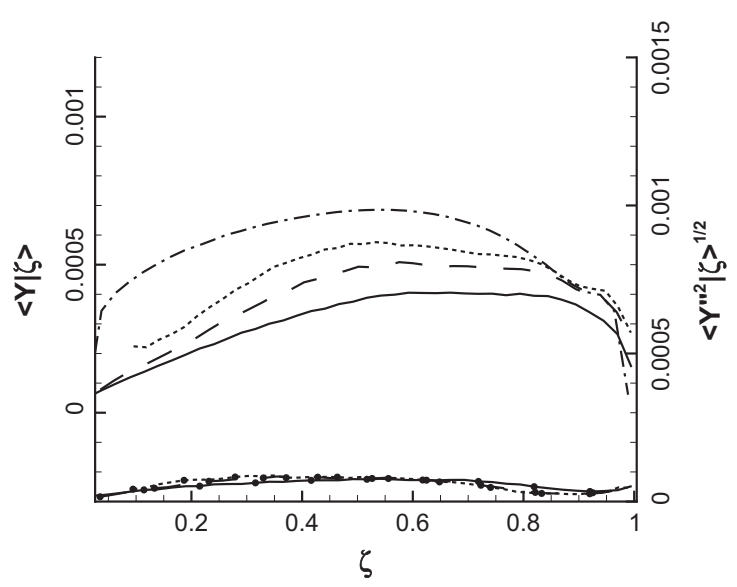

(a) $\mathrm{H}_{2}$

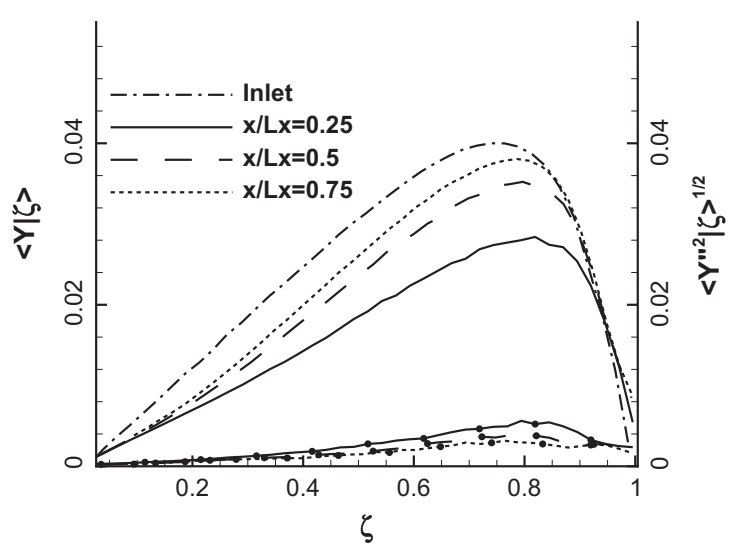

(b) $\mathrm{CO}$

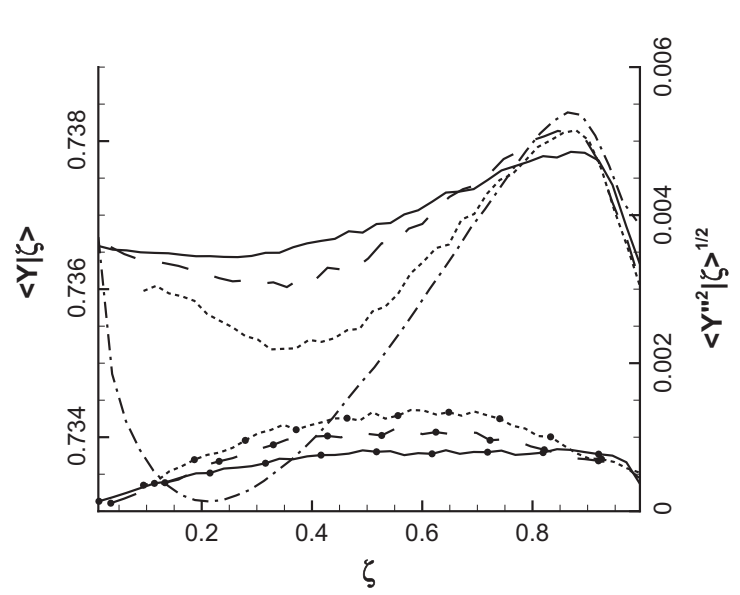

(c) $\mathrm{N}_{2}$

Fig. 8. Conditional means (lines) and rms values (lines with symbols) of $\mathrm{H}_{2}$, $\mathrm{CO}$, and $\mathrm{N}_{2}$ mass fractions in the Bunsen flame DNS at $x / L_{x}=0,0.25,0.5$, and 0.75 . curvature is explained by $\mathrm{H}_{2}$ molecules generated in the reaction zone diffusing towards the reactants faster than the progress variable. Note that in flamelet equations derived to include differential diffusion [50], the differential diffusion effect gives rise to a term involving convection in sample space which, in the absence of chemical reaction, is the only mechanism available to generate curvature of the kind seen in the $\mathrm{H}_{2}$ profiles for $\zeta<0.6$. Differential diffusion effects are also seen in the $\mathrm{N}_{2}$ profile in Fig. $8 \mathrm{c}$ where they

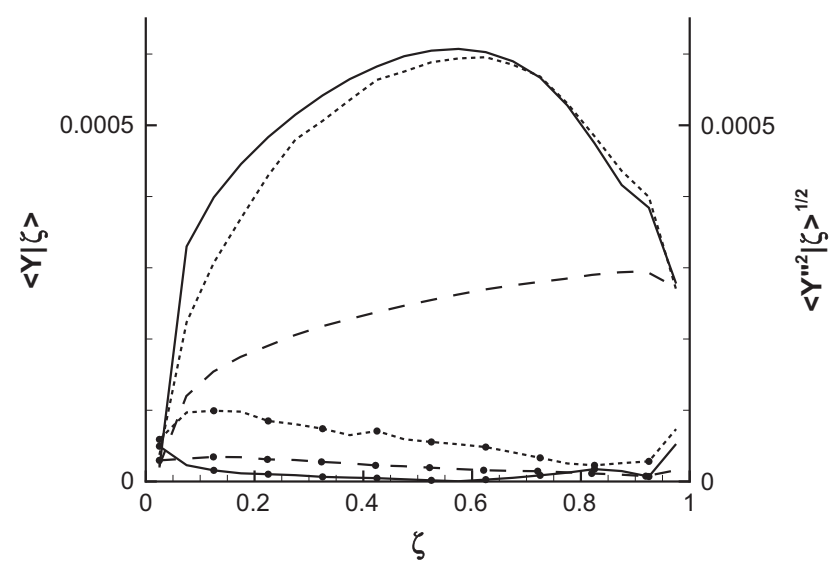

(a) $\mathrm{H}_{2}$

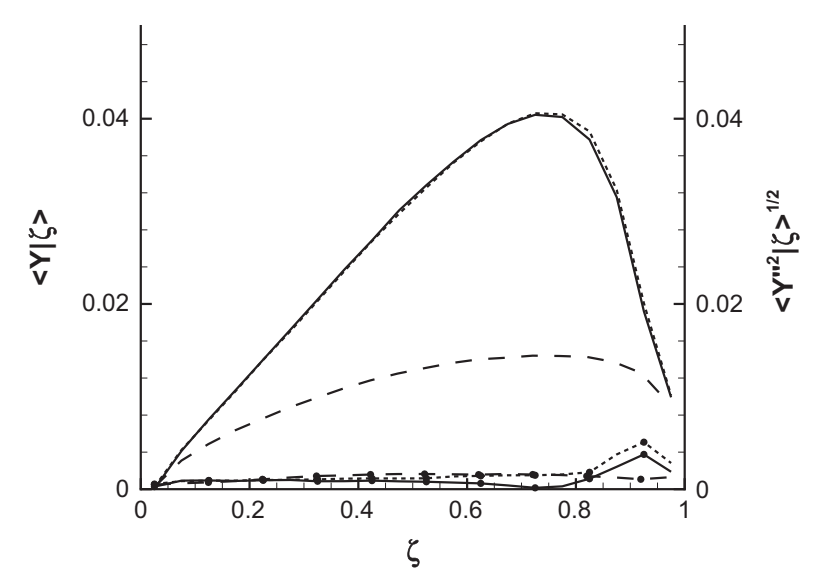

(b) $\mathrm{CO}$

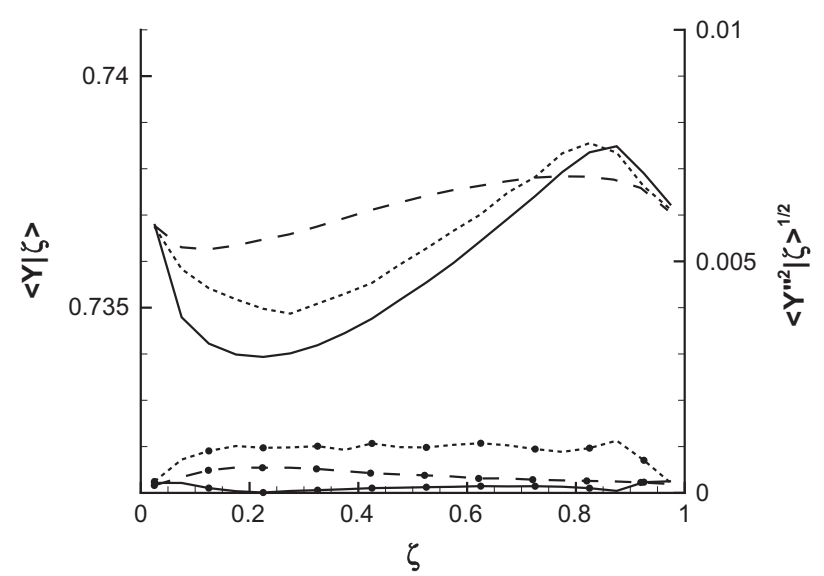

(c) $\mathrm{N}_{2}$

Fig. 9. Conditional means (lines) and rms values (lines with symbols) of $\mathrm{H}_{2}, \mathrm{CO}$, and $\mathrm{N}_{2}$ mass fractions in the 2D DNS: initial condition (lines); inert case at $t=\tau_{f}$ (dashes); and chemically reacting case at $t=\tau_{f}$ (dots). 
give a distinctive profile in progress variable space, thereby generating variance of $\mathrm{N}_{2}$ mass fraction. The molecular transport of $\mathrm{N}_{2}$ is seen to counteract the flux of highly mobile species, such as $\mathrm{H}_{2}$, from the reaction zone towards the unburned mixture. The $\zeta$-space curvature associated with differential diffusion is flattened by the intense turbulence in the near field of the jet but, like the flamelet structure, it recovers as the turbulence relaxes downstream.

The conditional mass fraction data for the 2D DNS cases in Fig. 9 show the same qualitative dependence on Damköhler number and differential diffusion as the 3D Bunsen case. This is despite the use of different molecular diffusion models (constant Lewis number and mixture averaged models respectively), and the different characteristics of the 3D and 2D turbulent flows. The conditionally averaged mass fraction profiles remain close to the initial laminar flame profile in the chemically reacting case, and they are in the process of relaxing towards a linear 'mixing only' profile in the inert, zero-Damköhler number case. In the chemically reacting 2D DNS, the persistence of the $\left\langle Y_{H_{2}} \mid \zeta\right\rangle$ curvature in the preheat zone $(\zeta<0.6)$, and of the $\left\langle Y_{N 2} \mid \zeta\right\rangle$ profile, are characteristic of differential diffusion and flamelet-regime combustion, as seen in the Bunsen flame DNS.

Reproducing the effects of differential diffusion and of the combustion regime which are seen in both the 3D Bunsen and 2D decaying turbulence DNS, in terms of their statistical effects on mixing rates and compositions, provides a challenging test of the ability of PDF mixing models to describe turbulent premixed combustion.

\subsection{Model performance}

The inert and chemically reacting 2D DNS cases have been simulated with the four mixing models: IEM, EMST, IEM-DD, and EMST-DD. We use only the 2D DNS configuration to compare these mixing models in this paper, but one-dimensional PDF simulations of the Bunsen flame - which support the conclusions of this paper are provided as supplementary material. The predicted evolution of the Favre mean progress variable is shown in Fig. 10 for the chemically reacting case (the Favre mean progress variable is constant in the non-reacting case and it is not shown). Each of the PDF mixing models under-predicts the rate of increase of the mean progress variable. The EMST models perform markedly better than the IEM model, while the respective differential diffusion corrections make relatively little difference to the mean progress variable in this case. The poor performance of the IEM models can be understood by examining the predicted progress variable PDFs at $t=\tau_{f}$, shown in Fig. 11. A well known deficiency of the IEM model is that, in the absence of chemical reaction or spatial transport, it preserves the

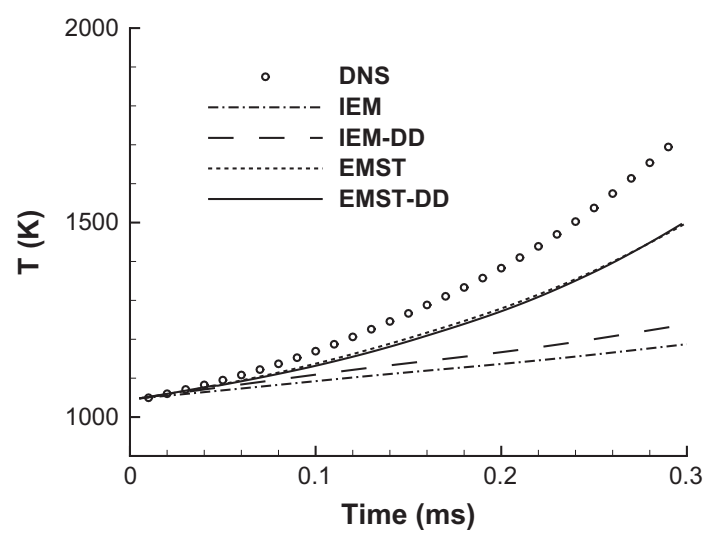

Fig. 10. Mean temperature evolution for the DNS, IEM, IEM-DD, EMST, and EMSTDD simulations. initial shape of the PDF leading to the poor predictions for the inert case in Fig. 11a. The EMST models, in contrast, predict the inert mixing of progress variable accurately. The superior predictions of progress variable mixing achieved by the EMST model can be attributed to the way it mixes locally in composition space, and therefore locally in progress variable space. In the chemically reacting case, the IEM models lead to strong under-prediction of the probability density within the reaction zone (implying that the IEM under-predicts the magnitude of progress variable's conditional diffusion rate in the reaction zone). The EMST models' predictions of the progress variable distribution are fairly close to the DNS distribution. Therefore the under-prediction of the mean progress variable by the EMST may be partly due to differences in the conditional statistics of minor species mass fractions presented below.

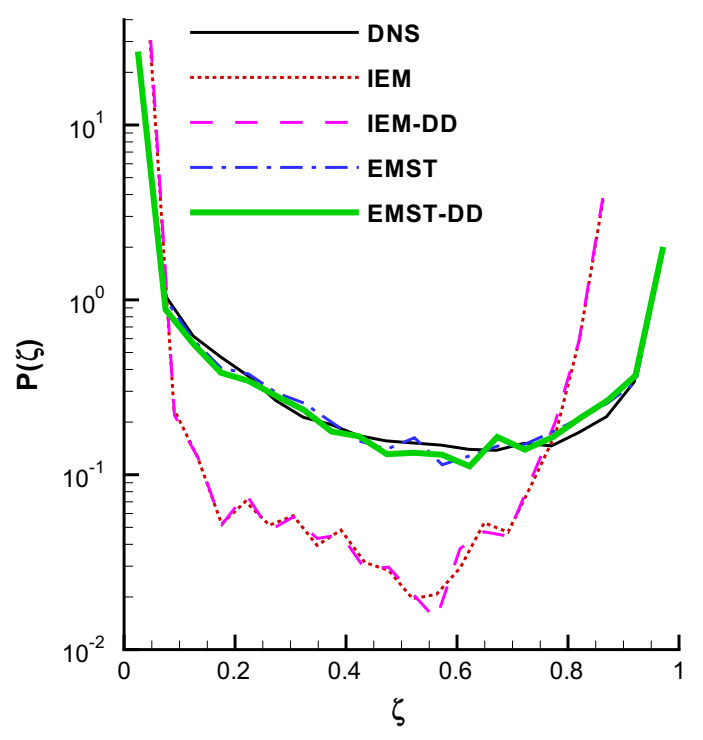

(a)

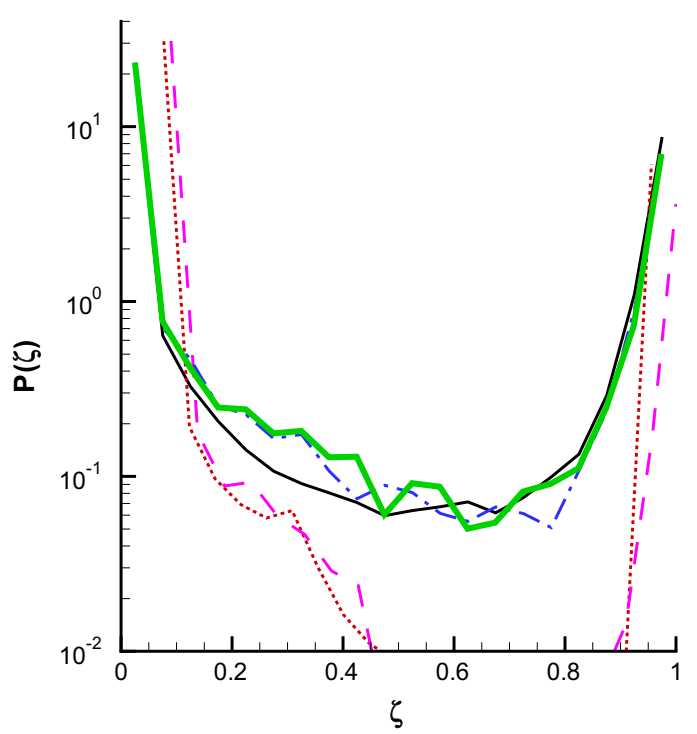

(b)

Fig. 11. Progress variable PDFs for the inert (top) and chemically reacting (bottom) cases at $t=\tau_{f}$. Data are for the DNS, and for PDF calculations with the IEM, IEM-DD, EMST, and EMST-DD models. 


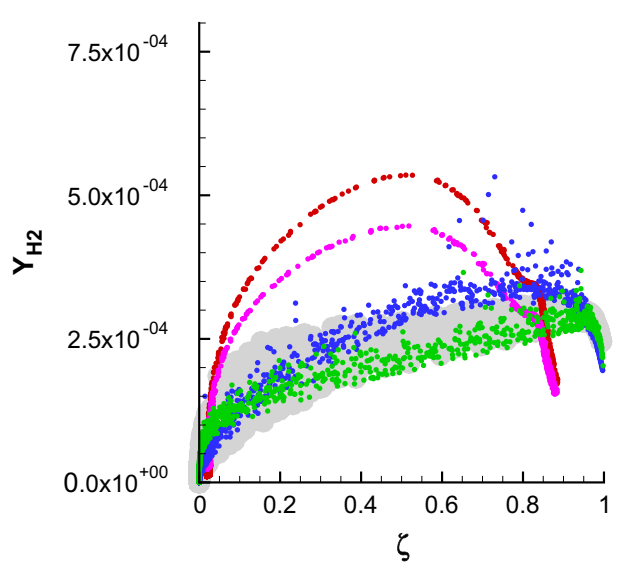

(a)

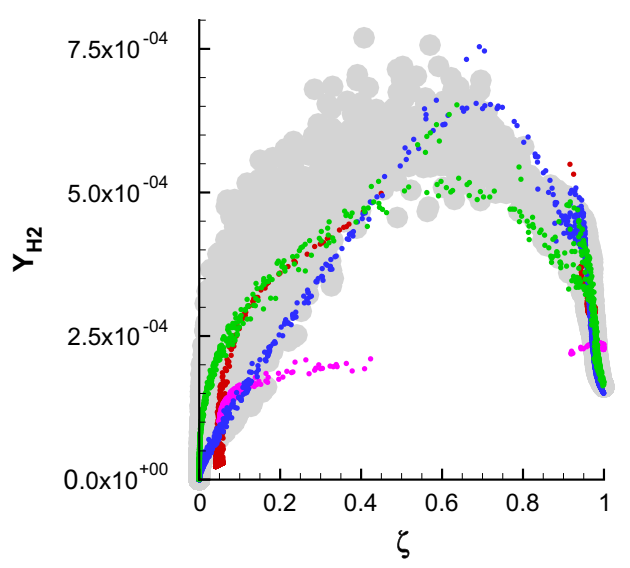

(c)

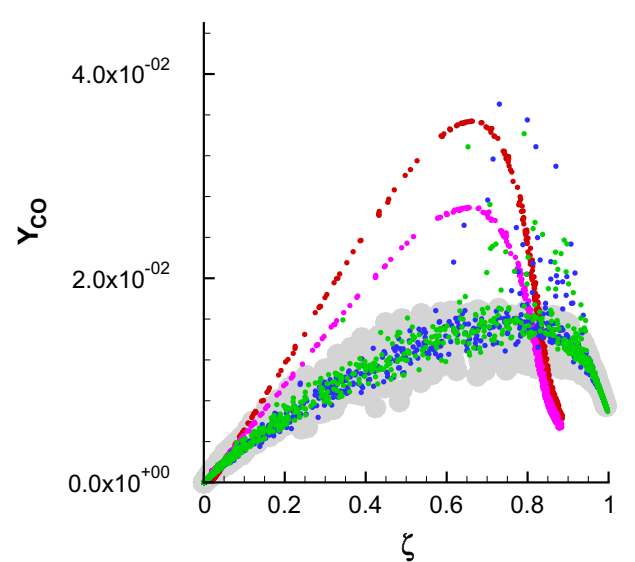

(b)

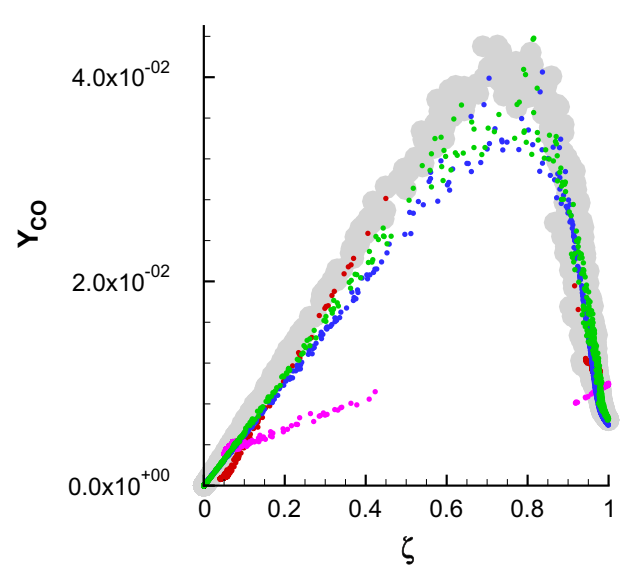

(d)

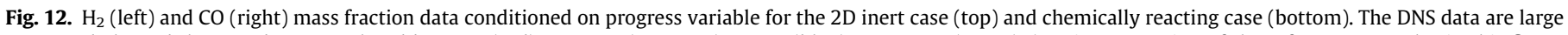

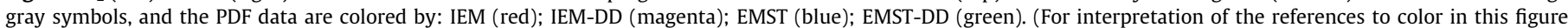
legend, the reader is referred to the web version of this article.)

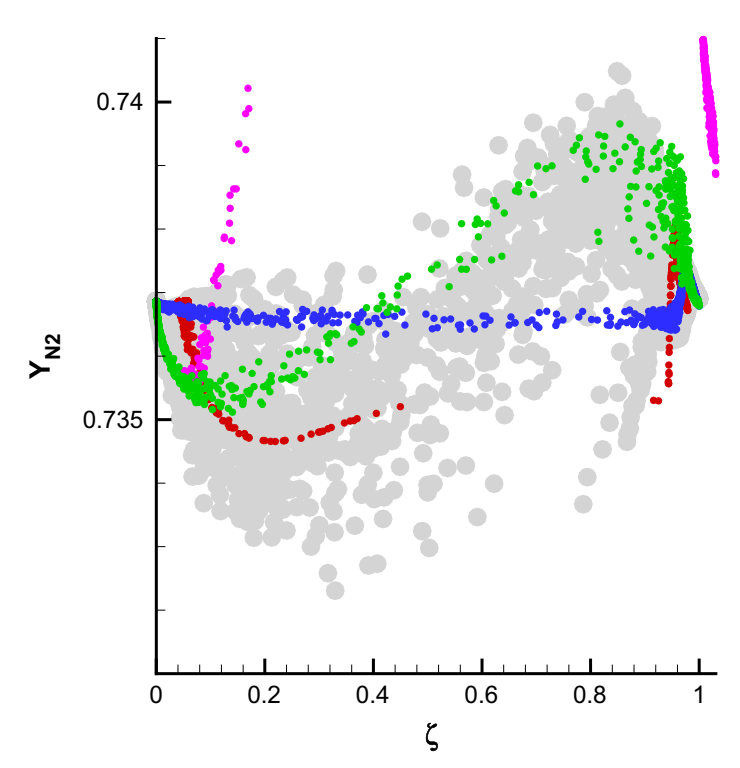

Fig. 13. $\mathrm{N}_{2}$ mass fraction data conditioned on progress variable for the $2 \mathrm{D}$ chemically reacting case. The data are colored as in Fig. 12. (For interpretation of the references to color in this figure legend, the reader is referred to the web version of this article.)

\subsubsection{Dissipation rate predictions}

The mixing rates $\left(\Lambda_{\alpha}\right)$ produced by the IEM model are strictly equal for all species. But the species mixing rates predicted by the EMST differ among species, reflecting the species' gradients in composition space. The variance decay rate ratios resulting from the EMST model, shown in Fig. $5 c$ and d, may be compared with the mixing rate ratios in the DNS, Fig. $5 a$ and $b$. The EMST model correctly shows an enhancement in the mixing rates of intermediate species relative to that of $\mathrm{O}_{2}$ (or progress variable). The magnitudes are not well predicted however. Predictions of the EMST-DD model with $C_{K}=1$ are given in Fig. 5e and f. Applying the differential diffusion correction to the EMST model leads to improved mixing rates, particularly of the highly diffusive species $\mathrm{H}_{2}$ and $\mathrm{H}$, which approach those observed in the reactive DNS. The EMST-DD model also reproduces the negative $\Lambda_{N 2}$ values (i.e. production of $\mathrm{N}_{2}$ variance) which occurs in the DNS due to differential diffusion.

Using $C_{K}=1$ improves predictions of the species mixing rate ratios for the chemically reacting case. For the inert case, $C_{K}=1$ predicts the initial mixing rate ratios well, while $C_{K}=0$ gives better predictions once the flamelet structure of the initial condition has dissipated. These observations support the earlier argument that, in turbulent premixed combustion, $C_{K}$ should transition from zero to unity when the Karlovitz number is of order unity. The model parameter $C_{K}$ can also be used to account for the influence of the initial (or boundary) conditions on the mixing process. The 
functional model for $C_{K}$ needs to be applicable across all combustion modes (i.e. diffusion flames, ignition fronts, propagating flames). The current study is restricted to premixed combustion and development of a general model for $C_{K}$ is left for future work.

\subsubsection{Composition statistics}

The conditional scatter of the $\mathrm{CO}$ and $\mathrm{H}_{2}$ mass fractions at $t=\tau_{f}$ are shown in Fig. 12, for the inert and chemically reacting DNS, and the respective PDF model predictions. In the inert case, the IEM model preserves the shape of the initial conditional composition distribution, leading to poor agreement with the DNS data. The IEM-DD model imposes the species scalar dissipation rates from the DNS (seen in Fig. 5a and b) which increases the mixing of both
$\mathrm{H}_{2}$ and CO. In the chemically reacting case, where the magnitudes of the mixing rate ratios are greater, the shape of the IEM-DD predictions differ from the DNS data. While the IEM-DD model imposes the correct scalar dissipation rates, it does so in a manner which does not respect scalar localness, resulting in unrealistic composition profiles.

Compared with the IEM model, the EMST predictions in Fig. 12 give better agreement with the DNS in both the inert and chemically reacting cases. The differential diffusion correction has little influence on the EMST-DD predictions of the conditional CO mass fraction distribution because its Lewis number is close to unity, but the shape of the $\mathrm{H}_{2}$ mass fraction profile is greatly improved and closely resembles the DNS.

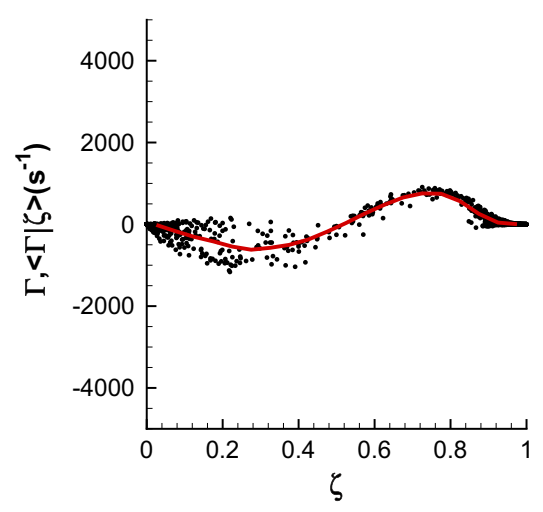

(a)

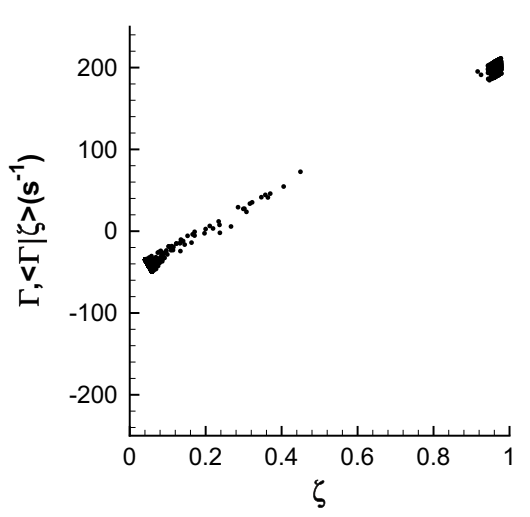

(d)

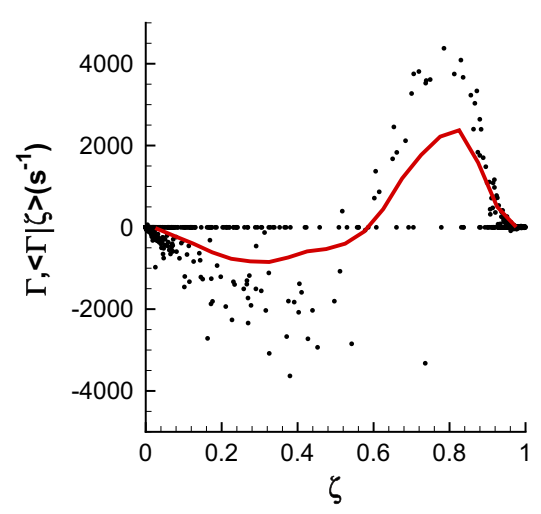

(g)

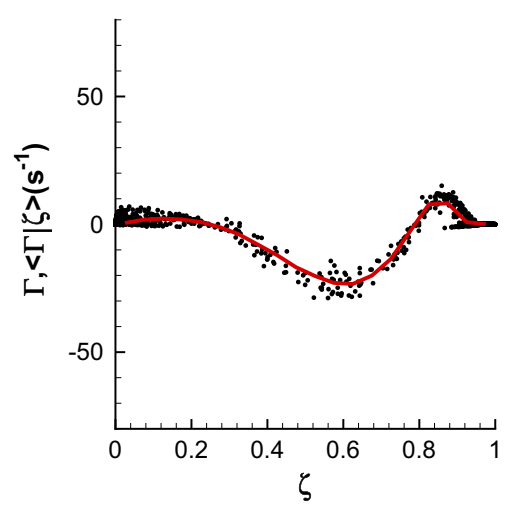

(b)

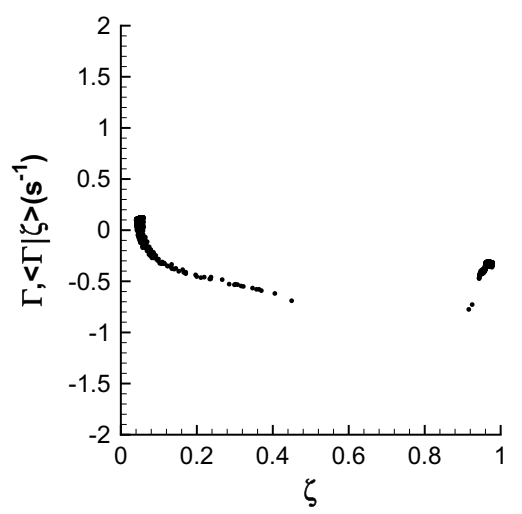

(e)

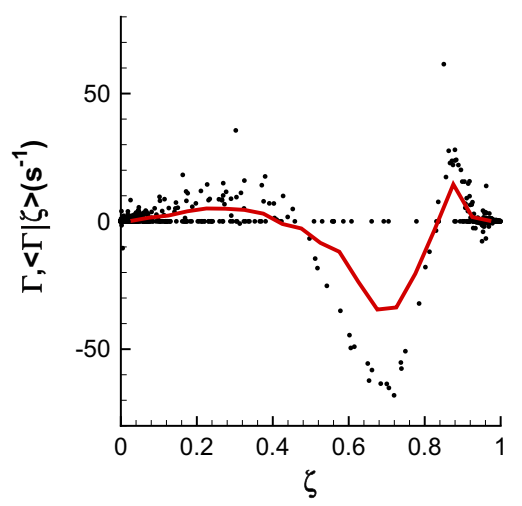

(h)

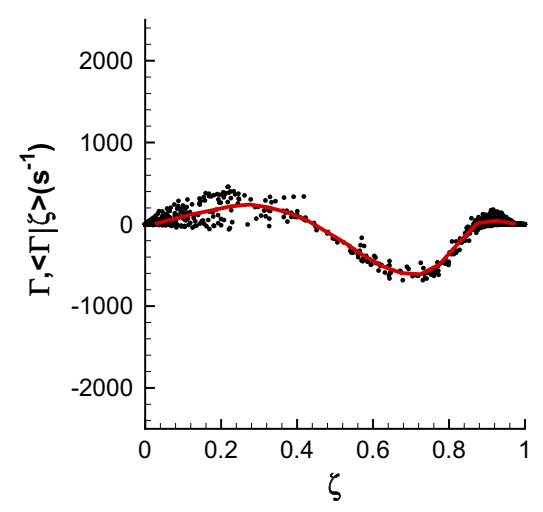

(c)

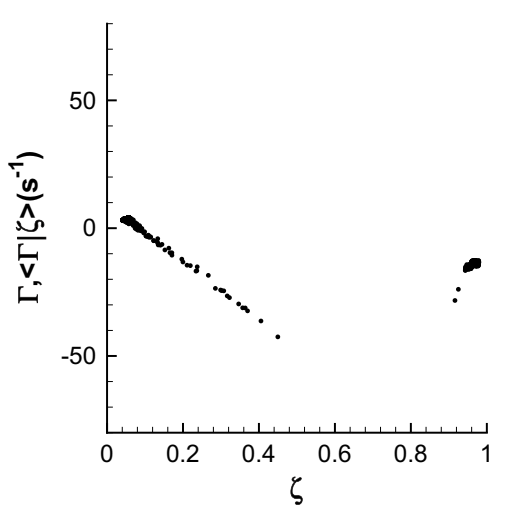

(f)

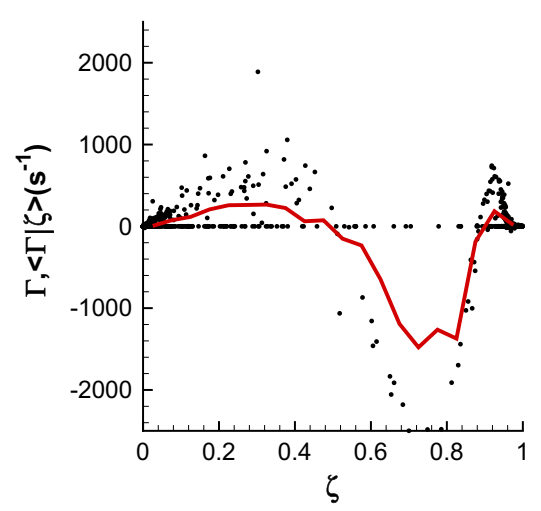

(i)

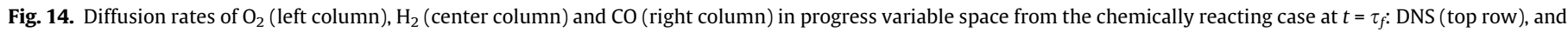
predictions using IEM (middle row), and EMST (bottom row). Lines are conditional means. 


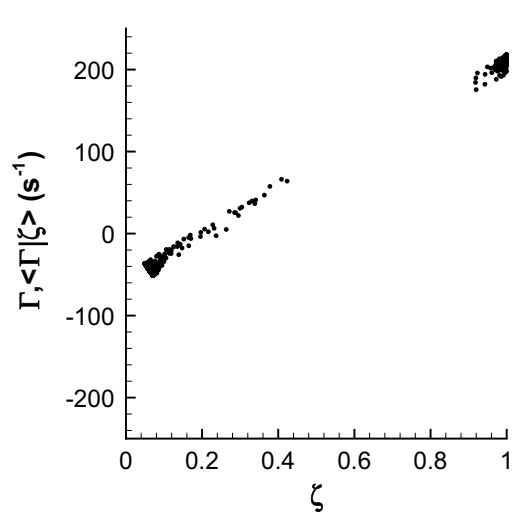

(a)

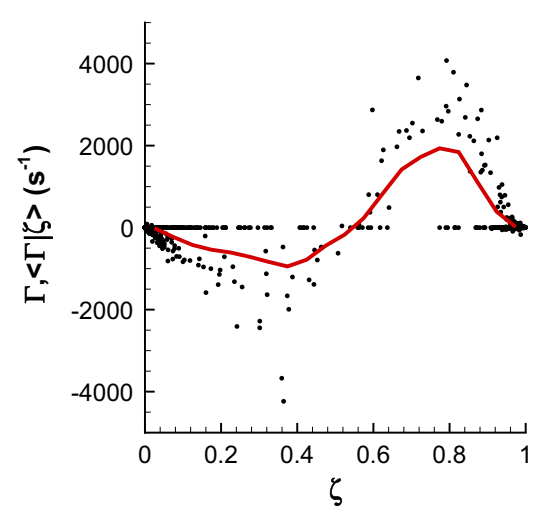

(d)

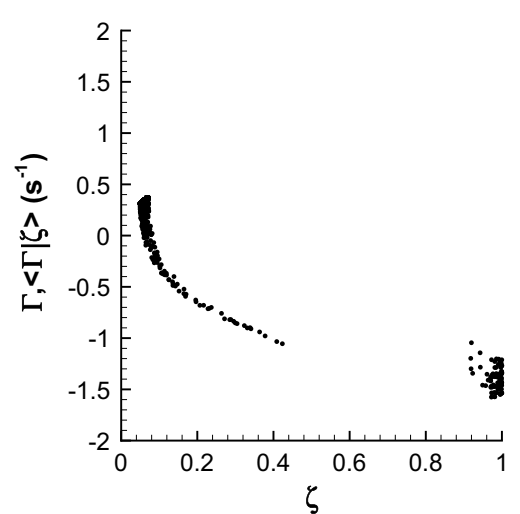

(b)

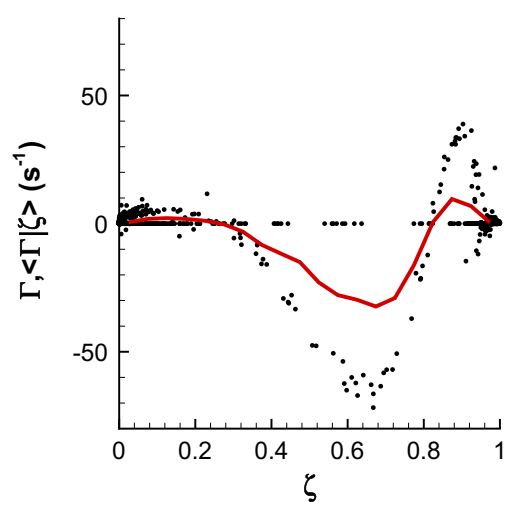

(e)

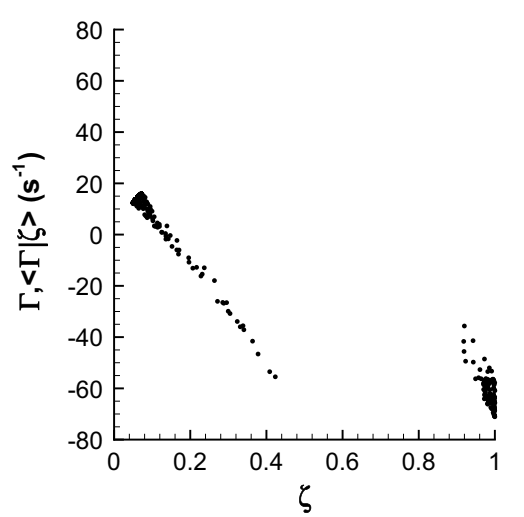

(c)

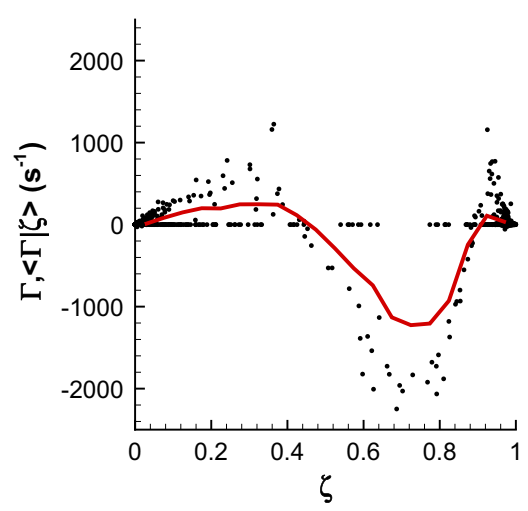

(f)

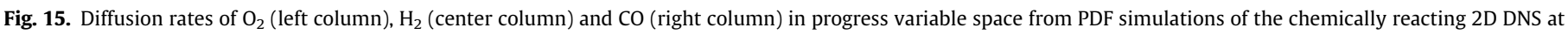
$t=\tau_{f}$ : IEM-DD (middle row), and EMST-DD (bottom row). Lines are conditional means.

Each of the mixing models under-predicts the degree of scatter around the conditional mean that occurs in the DNS calculations. Note that the PDF results in Fig. 12 comprise data from the 20 PDF simulations performed for each model. Because each PDF simulation starts with a different statistical sample of the DNS composition, each simulation produces slightly different results, and this contributes to the conditional scatter. The modelling of conditional variance production by the IEM and EMST models is discussed by Klimenko [51]. Klimenko shows that the standard IEM model does not contribute to generation of conditional fluctuations, and notes that the EMST model also tends to under-predict generation of conditional fluctuations. The under-prediction of conditional fluctuations by the EMST model is described as 'stranding' and, as noted by Meyer and Jenny [52], stranding persists despite the intermittent nature of mixing in the standard EMST model.

The distribution of $\mathrm{N}_{2}$ mass fraction across progress variable space is plotted in Fig. 13 for the chemically reacting case. The DNS data shows a distinct variation of the $\mathrm{N}_{2}$ mass fraction through the flame, which is only reproduced by the EMST-DD model. The EMST model produces a linear mixing profile, as should be expected in the absence of differential diffusion. Both of the IEM and IEM-DD models fail to predict the structure seen in the DNS data. The poor predictions of the IEM-DD model, in comparison to the EMST-DD predictions, illustrate the importance of including both differential mixing rates and localness for prediction of flamelet combustion.

Accounting for localness is less important when using the transported-PDF approach as a sub-grid model for highly-resolved LES (because the sub-grid variance is reduced). If satisfactory predictions of mixing can be achieved without accounting for localness, the IEM-DD model provides a means to describe differential diffusion effects at a lower computational cost than the EMST-DD model.

\subsubsection{Diffusion rate statistics}

Species diffusion rates from the chemically reacting 2D DNS are plotted versus progress variable in Fig. 14 (showing the DNS, IEM

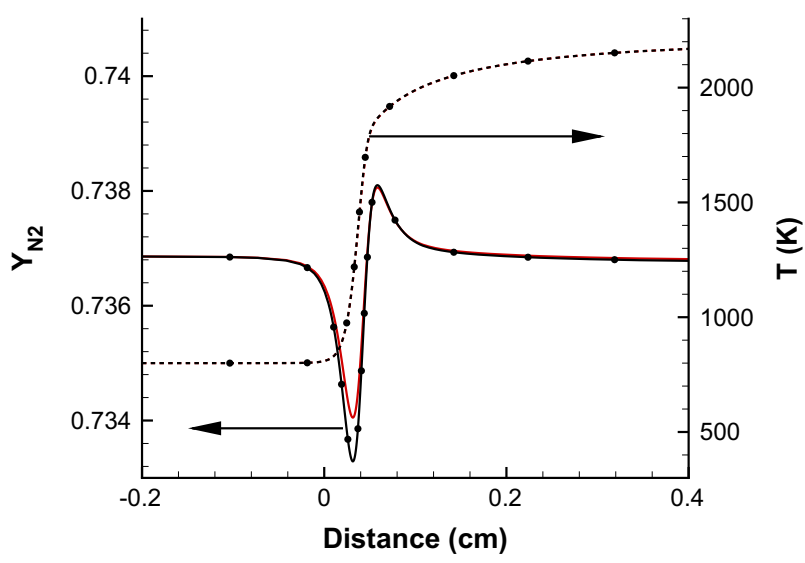

Fig. 16. Premixed laminar flame solutions for temperature (dots) and $Y_{\mathrm{N}_{2}}$ (lines) computed using mixture averaged (symbols) and multi-component (no-symbols) transport models. (The two temperature solutions are indistinguishable). 
and EMST results), and in Fig. 15 (showing the corresponding IEMDD and EMST-DD results). Note that $\Gamma=0$ for the particles that are excluded from mixing by the intermittency feature in the EMST models. The PDF results comprise data from 20 realizations. Due to limits of statistical convergence in the individual simulations, this introduces additional conditional scatter. This also reduces the appearance of 'stranding' which is more apparent when viewing the IEM and EMST simulations individually.

The shape of the diffusion rates predicted by the IEM model, Fig. 14, indicates that the IEM does not capture the physical structure of the mixing process occurring in the DNS. By accounting for localness, the EMST model reproduces the general pattern of the conditional diffusion rates observed in the DNS data. While the EMST model does reproduce the general pattern of the conditionally averaged diffusion rate, the conditional averages cross zero at higher values of progress variable than in the DNS.

The EMST-DD diffusion rate statistics for the reactive case are closer to the DNS data than for the standard EMST model: the locations where the conditionally averaged diffusion rates cross zero are similar to the DNS, and the peak magnitudes of the conditionally averaged diffusion rates remain similar to the predictions of the standard EMST model and of the DNS. It is interesting to note that while the peak magnitudes of the conditionally averaged diffusion rates are not greatly altered by the differential diffusion correction, the differential diffusion correction did produce an orderone change in the scalar dissipation rate ratios for several species (Fig. 5). Therefore the EMST-DD's improved prediction of scalar dissipation rates is not due to a wholesale increase or decrease in the species diffusion rates, but it is due to improved prediction of the composition's conditional statistics which gives good predictions for the shape of the conditionally averaged diffusion rates.

\section{Conclusions}

A general method for accounting for differential diffusion in pairwise-exchange mixing models has been developed in this paper, in a manner which satisfies realizability requirements. This method has been used to develop variants of the IEM and EMST mixing models which account for differential diffusion: the IEMDD and EMST-DD models respectively.

Two and three-dimensional DNS data for turbulent premixed methane-air combustion have been analyzed, and the 2D DNS data have been used to evaluate the performance of the PDF mixing models. The 2D and 3D DNS data both show that individual species mixing rates depend on the flame structure, and consequently on the species diffusivities, increasingly in high Damköhler number flows. The DNS also exhibits creation of $N_{2}$ mass fraction variance which has been explained through analysis of differential diffusion effects. The ability to predict these effects is an important measure of the suitability of PDF mixing models for premixed combustion applications exhibiting differential diffusion.

Simulations using the IEM and EMST models, and their differential diffusion variants, are compared with the 2D DNS data. The predictions of the EMST model are generally superior to the IEM model because it mixes in a manner which enforces localness, although it demands more computational effort. The differential diffusion correction in the EMST-DD model improves the predictions further, giving good predictions of the differences between scalar mixing rates, conditional statistics of the composition, and also capturing the production of $N_{2}$ mass fraction due to differential diffusion. Further investigation is required in order to develop general modelling for the EMST-DD model parameter $C_{K}$ across a range of combustion modes.

While the IEM-DD model can accommodate different mixing rates for every species, the differential diffusion correction modifies mixing in a way which is not related to the flame structure. If description of the flame structure is not critical to the mixing predictions, for example in sub-grid PDF modelling for highly-resolved LES, the IEM-DD model provides a method with potential to describe differential diffusion effects at a lower computational cost than the EMST-DD model.

\section{Acknowledgments}

Many constructive and detailed suggestions of three anonymous reviewers are gratefully acknowledged. This work was supported by the Division of Chemical Sciences, Geosciences and Bio-sciences, the Office of Basic Energy Sciences, the US Department of Energy. Sandia National Laboratories is a multiprogram laboratory operated by Sandia Corporation, a Lockheed Martin Company, for the US Department of Energy under contract DEAC04-94-AL85000. This research used resources of the National Center for Computational Sciences at Oak Ridge National Laboratory, which is supported by the Office of Science of the US Department of Energy under Contract No. DE-AC05-00OR22725; and E.S. Richardson received further support from the UK Engineering and Physical Sciences Research Council (Grant No. EP/I004564/1). In addition, the authors would like to thank Stephen B. Pope for helpful comments, and Fabrizio Bisetti for providing part of the PDF simulation program.

\section{Appendix A. Effect of differential diffusion on scalar variance}

This appendix demonstrates that differential diffusion can lead to generation of scalar variance. Consider a flow of a differentially diffusing mixture, in which the mass fraction of one species is initially homogeneous. We will show that differential diffusion introduces a variation of the species mass fraction that was initially homogeneous, thereby producing scalar variance. This can be demonstrated mathematically, or numerically. Two molecular transport models are considered: (1) mixture averaged transport and (2) multi-component transport.

The diffusive source term for the scalar variance of species $\alpha$ is $2 Y_{\alpha}^{\prime \prime} \Gamma_{\alpha}$ (see the variance transport equation, Eq. (B.2) in Ref. [9]). First, we consider the mixture-averaged transport model, which is based on the Curtiss-Hirschfelder approximation [53]. The diffusion rate is given by:

$\Gamma_{\alpha}=\Gamma_{\alpha}^{D}+\Gamma_{\alpha}^{C}$,

with the ordinary diffusion term,

$\rho \Gamma_{\alpha}^{D}=\nabla \cdot\left(\rho D_{\alpha} \frac{Y_{\alpha}}{X_{\alpha}} \nabla X_{\alpha}\right)$

and a correction term required to conserve mass in the case of nonequal diffusivities (p. 16 in Ref. [34]),

$\Gamma_{\alpha}^{C}=-Y_{\alpha} \sum_{\beta} \Gamma_{\beta}^{D}$.

The contributions of ordinary diffusion and the correction term can be written separately:

$2 \widetilde{Y_{\alpha}^{\prime \prime} \Gamma_{\alpha}^{D}}+2 \widetilde{Y_{\alpha}^{\prime \prime} \Gamma_{\alpha}^{C}}$.

$Y_{\alpha}^{\prime \prime}$ and $\Gamma_{\alpha}^{D}$ are negatively correlated (above average values of the mass fraction typically result in a negative diffusive flux), so the ordinary diffusion causes a reduction of the scalar variance. The contribution of the correction term however can have either sign. In situations where the correction term has a greater magnitude than the ordinary diffusion term it is possible for differential diffusion to result in an increase in individual scalar variances. 
Where a premixed flame propagates through an initially homogeneous mixture, any variation of an inert scalar mass fraction must be due to molecular transport. Figure 16 presents temperature and nitrogen mass fraction profiles through a freely propagating planar laminar premixed flame, simulated with mixture averaged and multi-component transport models in the CHEMKIN III software [47]. The flame propagates into an unburnt mixture of methane-air with equivalence ratio of 0.7 , temperature of $800 \mathrm{~K}$, and pressure of $1 \mathrm{~atm}$. The transport properties and the thermochemistry are modelled using the GRI-3.0 natural gas combustion mechanism with all chemical reactions involving nitrogen deactivated. Highly resolved, grid-independent solutions demonstrate that both the mixture averaged and the multi-component transport models predict that differential diffusion generates scalar variation of the $\mathrm{N}_{2}$ mass fraction.

\section{Appendix B. Effect of differential diffusion on particle velocity}

In models where the PDF-particle weights vary due to differential diffusion, particle momentum and kinetic energy also change. A corrective acceleration term has been derived in order to enforce pairwise conservation of momentum and energy during the mixing process, and this correction term should be added to the acceleration terms in the PDF particle transport model. For pairwise mixing between particles $p$ and $q$, the rate of change of the velocities $d \mathbf{u}^{(p)} / d t$ and $d \mathbf{u}^{(q)} / d t$, can be determined by enforcing the pairwise conservation of mass

$\left.\frac{d w^{(p)}}{d t}\right|_{q}+\left.\frac{d w^{(q)}}{d t}\right|_{p}=0$

of momentum

$\left.\frac{d w^{(p)} \mathbf{u}^{(p)}}{d t}\right|_{q}+\left.\frac{d w^{(q)} \mathbf{u}^{(q)}}{d t}\right|_{p}=0$

and of kinetic energy

$\left.\frac{d w^{(p)} \mathbf{u}^{(p) 2}}{d t}\right|_{q}+\left.\frac{d w^{(q)} \mathbf{u}^{(q) 2}}{d t}\right|_{p}=0$.

Expanding Eq. (B.2) using the product rule, and using conservation of mass gives

$\left.\left(\mathbf{u}^{(p)}-\mathbf{u}^{(q)}\right) \frac{d w^{(p)}}{d t}\right|_{q}+\left[\left.w^{(p)} \frac{d \mathbf{u}^{(p)}}{d t}\right|_{q}+\left.w^{(q)} \frac{d \mathbf{u}^{(q)}}{d t}\right|_{p}\right]=0$,

and, after further rearrangement

$\left.\frac{d \mathbf{u}^{(p)}}{d t}\right|_{q}=-\left[\left.\left(\mathbf{u}^{(p)}-\mathbf{u}^{(q)}\right) \frac{d w^{(p)}}{d t}\right|_{q}+\left.w^{(q)} \frac{d \mathbf{u}^{(q)}}{d t}\right|_{p}\right] / w^{(p)}$.

Rearrangement of Eq. (B.3) using the product rule and conservation of mass gives:

$\left.\left(\mathbf{u}^{(p) 2}-\mathbf{u}^{(q) 2}\right) \frac{d w^{(p)}}{d t}\right|_{q}+\left[\left.w^{(p)} \frac{d \mathbf{u}^{(p) 2}}{d t}\right|_{q}+\left.w^{(q)} \frac{d \mathbf{u}^{(q) 2}}{d t}\right|_{p}\right]=0 ;$

and then applying the chain rule:

$\left(\mathbf{u}^{(p) 2}-\mathbf{u}^{(q) 2}\right) \frac{d w^{(p)}}{d t}+\left[2 w^{(p)} \mathbf{u}^{(p)} \frac{d \mathbf{u}^{(p)}}{d t}+2 w^{(q)} u^{(q)} \frac{d \mathbf{u}^{(q)}}{d t}\right]=0$.

Substituting the expression in Eq. (B.5) for $d \mathbf{u}^{(p)} /\left.d t\right|_{q}$ gives

$\left.\left(\mathbf{u}^{(p) 2}-\mathbf{u}^{(q) 2}\right) \frac{d w^{(p)}}{d t}\right|_{q}=2 \mathbf{u}^{(p)}\left[\left.\left(\mathbf{u}^{(p)}-\mathbf{u}^{(q)}\right) \frac{d w^{(p)}}{d t}\right|_{q}+\left.w^{(q)} \frac{d \mathbf{u}^{(q)}}{d t}\right|_{p}\right]$

$-\left.2 \mathbf{u}^{(q)} w^{(q)} \frac{d \mathbf{u}^{(q)}}{d t}\right|_{p}$ which leads to an expression for the rate of change of velocity $\mathbf{u}^{(q)}$ :

$$
\begin{aligned}
\left.\frac{d \mathbf{u}^{(q)}}{d t}\right|_{p} & =-\left.\frac{\left(\mathbf{u}^{(p) 2}-2 \mathbf{u}^{(p)} \mathbf{u}^{(q)}+\mathbf{u}^{(q) 2}\right)}{2 w^{(q)}\left(\mathbf{u}^{(p)}-\mathbf{u}^{(q)}\right)} \frac{d w^{(p)}}{d t}\right|_{q} \\
& =\left.\frac{\left(\mathbf{u}^{(p)}-\mathbf{u}^{(q)}\right)}{2 w^{(q)}} \frac{d w^{(q)}}{d t}\right|_{p} ;
\end{aligned}
$$

and similarly for $\mathbf{u}^{(p)}$ :

$$
\left.\frac{d \mathbf{u}^{(p)}}{d t}\right|_{q}=\left.\frac{\left(\mathbf{u}^{(q)}-\mathbf{u}^{(p)}\right)}{2 w^{(p)}} \frac{d w^{(p)}}{d t}\right|_{q}=\left.\frac{w^{(q)}}{w^{(p)}} \frac{d \mathbf{u}^{(q)}}{d t}\right|_{p}
$$

\section{Appendix C. The variance equation for the IEM-DD model}

The variance equation for the IEM-DD model (Eqs. 14 and 15) is derived as follows. First, note that the IEM model leaves the mean mass fraction expectations unchanged,

$\frac{d E\left\{Y_{\alpha}^{\prime \prime 2}\right\}}{d t}=\frac{d E\left\{Y_{\alpha}^{2}\right\}}{d t}$

with,

$E\left\{Y_{\alpha}^{2}\right\}=\frac{\sum_{i=1}^{N} w^{(i)} Y_{\alpha}^{(i) 2}}{\sum_{i=1}^{N} w^{(i)}}$

Differentiating Eq. (C.2) with respect to time and applying the quotient rule gives,

$\frac{d E\left\{Y_{\alpha}^{2}\right\}}{d t}=\frac{d / d t\left\{\sum_{i=1}^{N} w^{(i)} Y_{\alpha}^{(i) 2}\right\}}{\sum_{i=1}^{N} w^{(i)}}-\frac{\sum_{i=1}^{N} w^{(i)} Y_{\alpha}^{(i) 2}}{\sum_{i=1}^{N} w^{(i)}} \frac{\sum_{i=1}^{N} d w^{(i)} / d t}{\sum_{i=1}^{N} w^{(i)}}$.

Since the sum of the particle weights is conserved, the second term on the right hand side is zero. Expanding the remaining term using the product and chain rules,

$\frac{d E\left\{Y_{\alpha}^{2}\right\}}{d t}=\sum_{i=1}^{N}\left\{2 w^{(i)} Y_{\alpha}^{(i)} \frac{d Y_{\alpha}^{(i)}}{d t}+Y_{\alpha}^{(i) 2} \frac{d w^{(i)}}{d t}\right\} / \sum_{i=1}^{N} w^{(i)}$

and using Eqs. 14 and 15 gives:

$\frac{d E\left\{Y_{\alpha}^{2}\right\}}{d t}=-\sum_{i=1}^{N} w^{(i)} Y_{\alpha}^{(i)}\left\{2 \frac{C_{\alpha} \omega}{2}\left(Y_{\alpha}^{(i)}-\left\langle Y_{\alpha}\right\rangle\right)-Y_{\alpha}^{(i)} \sum_{\beta=1}^{M} \frac{C_{\beta} \omega}{2}\left(Y_{\beta}^{(i)}-\left\langle Y_{\beta}\right\rangle\right)\right\} / \sum_{i=1}^{N} w^{(i)}$.

Noting that the first term on the right hand side involves the variance, and substituting Eq. (15) into the last term, the variance source term can be written as:

$\frac{d E\left\{Y_{\alpha}^{2}\right\}}{d t}=-C_{\alpha} \omega E\left\{Y_{\alpha}^{\prime \prime 2}\right\}+\frac{\sum_{i=1}^{N}\left(Y_{\alpha}^{(i)}\right)^{2} \frac{d w^{(i)}}{d t}}{\sum_{i=1}^{N} w^{(i)}}$.

When the species mixing coefficients are the same for each species the final term in Eq. (C.6) is zero, and the variance equation for the standard IEM model is recovered. Because $d w^{(i)} / d t$ can be positive, the IEM-DD model can lead to production of scalar variance.

\section{Appendix D. Supplementary data}

Supplementary data associated with this article can be found, in the online version, at doi:10.1016/j.combustflame.2012.02.026.

\section{References}

[1] C. Dopazo, E.E. O’Brien, Acta Astronaut. 1 (1974) 1239-1266.

[2] C. Dopazo, E.E. O’Brien, Phys. Fluids 17 (1985) 1968-1975. 
[3] S.B. Pope, Prog. Energy Combust. Sci. 11 (1985) 119-192.

[4] A.T. Norris, S.B. Pope, Combust. Flame 83 (1991) 27-42.

[5] S. Subramaniam, S.B. Pope, Combust. Flame 115 (1998) 487-514.

[6] M.J. Cleary, A.Y. Klimenko, J. Janicka, M. Pfitzner, Proc. Combust. Inst. 32 (2009) 1499-1507.

[7] J.-Y. Chen, W.-C. Chang, Combust. Sci. Technol. 133 (4) (1998) 343-375.

[8] D.W. Meyer, Phys. Fluids 22 (2010) 035103.

9] D.C. Haworth, Prog. Energy Combust. Sci. 36 (2) (2010) 168-259.

[10] R. McDermott, S.B. Pope, J. Comput. Phys. 226 (2007) 947-993.

[11] O. Zeman, J.L. Lumley, J. Atmos. Sci. 33 (1976) 1974-1988.

[12] W.P. Jones, P. Musonge, Phys. Fluids 31 (1988) 3589-3604.

[13] T. Mantel, R. Borghi, Combust. Flame 96 (4) (1994) 443-457.

[14] N. Swaminathan, K.N.C. Bray, Combust. Flame 143 (4) (2005) 549-565.

[15] A. Mura, R. Borghi, Combust. Flame 133 (1-2) (2003) 193-196.

[16] T.S. Kuan, R.P. Lindstedt, E.M. Vaos, in: G. Roy (Ed.), Advances in Confined Detonations and Pulse Detonation Engines, Torus Press, Moscow, 2003, pp. 1740.

[17] R.P. Lindstedt, E.M. Vaos, Combust. Flame 145 (2006) 495-511.

[18] E.R. Hawkes, R. Sankaran, J.C. Sutherland, J.H. Chen, Proc. Combust. Inst. 31 (2007) 16331640.

[19] E.S. Richardson, R. Sankaran, R.W. Grout, J.H. Chen, Combust. Flame 157 (3) (2010) 506-515

[20] A. Juneja, S.B. Pope, Phys. Fluids 8 (1996) 2161-2184.

[21] P.K. Yeung, S.B. Pope, Phys. Fluids A 5 (1993) 2467-2478.

[22] P.K. Yeung, J. Fluid Mech. 321 (1996) 235-278.

[23] P.K. Yeung, Phys. Fluids 10 (1998) 2621-2635.

[24] P.K. Yeung, M.C. Sykes, P. Vedula, Phys. Fluids 12 (2000) 1601-1604.

[25] N. Peters, Turbulent Combustion, Cambridge University Press, Cambridge, 2000.

[26] C. Dopazo, Phys. Fluids A 18 (4) (1975) 397-404.

[27] J. Janicka, W. Kolbe, W. Kollmann, J. Nonequil. Thermodynam. 4 (1979) 47-66.

[28] H. Chen, S. Chen, R.H. Kraichnan, Phys. Rev. Lett. 63 (1989) 2657-2660.

[29] S.B. Pope, Theor. Comput. Fluid Dynam. 2 (1991) 255-270.

[30] K. Vogiatzaki, A. Kronenburg, S. Navarro-Martinez, W.P. Jones, Proc. Combust. Inst. 33 (1) (2011) 1523-1531.

[31] L. Valiño, C. Dopazo, Phys. Fluids A 3 (1991) 3034-3037.
[32] R.O. Fox, Phys. Fluids 6 (1994) 334-348.

33] D.W. Meyer, P. Jenny, Phys. Fluids 18 (2006) 035105

[34] T. Poinsot, D. Veynante, Theoretical and Numerical Combustion, Second ed. R.T. Edwards Inc., Philadelphia, 2005.

[35] R. Barlow, M. Dunn, M. Sweeney, S. Hochgreb, in: Proceedings of the 10th International Workshop on Measurement and Computation of Turbulent (Non)premixed Flame, Beijing, China, July 29-31, 2010, pp. 253-254.

[36] S. Fedotov, M. Imhe, H. Pitsch, Phys. Rev. E 75 (2005) 016310.

[37] Jayesh, S.B. Pope, Technical Report No. FDA 95-05, Cornell University, 1995

[38] S.B. Pope, Turbulent Flows, Cambridge University Press, Cambridge, 2000.

[39] Z. Ren, S. Subramaniam, S.B. Pope, Implementation of the EMST Mixing Model, 2002. <http://eccentric.mae.cornell.edu/tcg/emsts>

[40] F. Bisetti, J.Y. Chen, E.R. Hawkes, J.H. Chen, Combust. Flame 155 (2008) 571584.

[41] R. Sankaran, E.R. Hawkes, C.S. Yoo, J.H. Chen, T. Lu, C.K. Law, in: 5th US National Combustion Meeting, 2007, B09.

[42] T. Passot, A. Pouquet, J. Fluid Mech. 181 (1987) 441-466

[43] C.A. Kennedy, M.H. Carpenter, Appl. Numer. Math. 14 (4) (1994) 397-433.

[44] C.A. Kennedy, M.H. Carpenter, R.M. Lewis, Appl. Numer. Math. 35 (2000) 177219.

[45] F. Frenklach, H. Wang, C.-L. Yu, M. Goldenberg, C.T. Bowman, R.K. Hanson, D.F. Davidson, E.J. Chang, G.P. Smith, D.M. Golden, W.C. Gardiner, V. Lissianski, $<$ http://me.berkeley.edu/gri_mech>.

[46] R. Sankaran, E.R. Hawkes, J.H. Chen, T. Lu, C.K. Law, Proc. Combust. Inst. 31 (1) (2007) 1291-1298.

[47] R.J. Kee, F.M. Rupley, E. Meeks, J.A. Miller, Report No. SAND96-8216, Sandia National Laboratories, 1996.

[48] R.J. Kee, G. Dixon-Lewis, J. Warnatz, M.E. Coltrin, J.A. Miller, Report No. SAND86-8246, Sandia National Laboratories, 1986.

[49] J.D. Lambert, Numerical Methods for Ordinary Differential Systems: The Initial Value Problem, Wiley, 1991.

[50] H. Pitsch, N. Peters, Combust. Flame 114 (1998) 26-40.

[51] A.Y. Klimenko, Combust. Flame 143 (2005) 369-385.

[52] D.W. Meyer, P. Jenny, J. Comput. Phys. 228 (2009) 12751293.

[53] C.F. Curtiss, H.O. Hirschfelder, J. Chem. Phys. 17 (1949) 550-555. 


\title{
Application of PDF mixing models to premixed flames with differential diffusion - Supplementary material
}

\author{
E.S. Richardson ${ }^{1, *}$, J.H. Chen ${ }^{2}$ \\ ${ }^{1}$ University of Southampton, Faculty of Engineering and the Environment, University Road, Southampton, SO17 \\ 1BJ, UK, e.s.richardson@ soton.ac.uk \\ ${ }^{2}$ Sandia National Laboratories, Combustion Research Facility, PO Box 969, Livermore, California, 94551-0969, \\ USA, jhchen@sandia.gov
}

\begin{abstract}
This supplementary material reports additional PDF modelling and analysis of a 3D turbulent premixed Bunsen flame. The EMST-DD model is again seen to improve predictions of differential diffusion in premixed flames, providing additional support to the conclusions of the main paper, which were based on 2D decaying turbulence.
\end{abstract}

\section{Simulation configuration and methods}

Turbulent flame DNS: A three-dimensional turbulent perfectly-premixed Bunsen flame has been analyzed. The flame simulated by Sankaran et al. [1] comprises a planar jet of unburned methane and air at $800 \mathrm{~K}, 1 \mathrm{~atm}$ and equivalence ratio $\phi=0.7$ issuing into a coflowing product stream from adiabatic combustion of the mixture. The DNS configuration is shown in Fig. 1 of the main paper. The slot jet width, $\mathrm{H}=1.8 \mathrm{~mm}$, the jet velocity $\left(100 \mathrm{~ms}^{-1}\right)$ and coflow velocity $\left(25 \mathrm{~ms}^{-1}\right)$ give a jet Reynolds number of 2100. The simulations were performed using the DNS code S3D, using a reduced chemical reaction model with 13 species [5], and constant, non-unity Lewis number transport (except for $\mathrm{N}_{2}$ which makes up the balance of the composition). Full details of the simulation are presented by Richardson et al. [2].

PDF simulation method: The premixed Bunsen configuration has also been simulated using a one-dimensional transported PDF approach, implemented using Lagrangian particles. The PDF calculations use the mean velocity field, the turbulent kinetic energy, and the Favre averaged dissipation rate taken from the DNS. The PDF computations exploit the statistical homogeneity along the $\mathrm{z}$ direction, and symmetry around the $y=0$ plane in the DNS configuration. Further simplification is made by employing a one-dimensional PDF domain (which extends across half of the y-direction in the DNS domain), and integrating the PDF equations in spatial increments along the $\mathrm{x}$ direction (by assuming that the time increments $\Delta t=$ $\Delta \mathrm{x} / \tilde{\mathrm{U}}$, where $\tilde{\mathrm{U}}$ is the mean axial velocity from the DNS). The cross-stream positions of the particles are advanced using the simplified Langevin model for particle acceleration [3]. This simple parabolic solution method cannot be expected to give accurate predictions of the mean flame shape in general. Here we report conditional statistics, and ratios of mixing timescales, which are relatively insensitive to the overall flame shape, and which permit a useful comparison between micro-mixing models. PDF simulation data are presented for the IEM, EMST, and

*Corresponding author: e.s.richardson@ soton.ac.uk Proceedings of the European Combustion Meeting 2011
EMST-DD models. The PDF simulation is initialized at $0.25 \mathrm{~L}_{\mathrm{x}}$ jet heights from the nozzle, and the results are reported at $0.5 \mathrm{~L}_{\mathrm{x}}$.

\section{Results and Discussion}

Ratios of $\Lambda_{\alpha} / \Lambda_{\mathrm{O}_{2}}$ for $\alpha \in\left\{\mathrm{H}_{2}, \mathrm{H}, \mathrm{OH}, \mathrm{CO}, \mathrm{N}_{2}\right\}$ are also shown for the EMST and EMST-DD models in Fig. 1. Note that the IEM model gives identical $\Lambda_{\alpha}$ for all species, and is not plotted. The EMST model predicts mixing ratios which are close to unity at the centre of the flame brush $(\mathrm{y} / \mathrm{H} \approx 0.5)$. The EMST-DD model gives greatly improved prediction of the relative species mixing rates. The EMST-DD data are shown for $\mathrm{C}_{\mathrm{K}}=0.3$ which gives close agreement with the DNS mixing rates.

Diffusion rates of $\mathrm{Y}_{\mathrm{CO}}$, sampled from across the $\mathrm{y}$ direction at $\mathrm{x}=0.5 \mathrm{~L}_{\mathrm{x}}$, are plotted in Fig. 2 for DNS, IEM, and EMST simulations. Data are plotted versus the progress variable sample space variable $\zeta$, where progress variable (equal to zero in reactants and unity in the products) has been based on $Y_{O 2}$. The IEM model fails to predict the structure of the diffusion process in progress variablespace. The EMST model gives a distribution of mixing rates which have the same shape as the DNS. The frequent occurrence of zero mixing in the EMST is due to the model's intermittency feature described in [4]. The species dependent mixing coefficients then serve to adjust the relative magnitude of the species mixing terms, resulting in the improved predictions of the mixing rate ratios seen in Fig. 1.

Differential diffusion is responsible for the curvature of the conditional mean $\mathrm{Y}_{\mathrm{H}_{2}}$ profile, in the non-reactive mixture of the pre-heat zone $(\zeta<0.5)$, which is seen for the DNS data in Fig. 3a. Since there is no chemical reaction in this mixture, the curvature arises because $\mathrm{Y}_{\mathrm{H}_{2}}$ diffuses faster into the reactants than the diffusion of progress variable itself. Over the same range of $\zeta$, the EMST model predicts a linear variation of the conditional mean. The EMST-DD, however, produces accurate predictions of the conditional mean and rms of $\mathrm{Y}_{\mathrm{H}_{2}}$ for $\zeta<0.5$. The conditionally averaged $\mathrm{Y}_{\mathrm{H}_{2}}$ diffusion rates in Fig. $3 \mathrm{~b}$ also shows that the EMST-DD model improves upon the predictions of the standard EMST. EMST pre- 

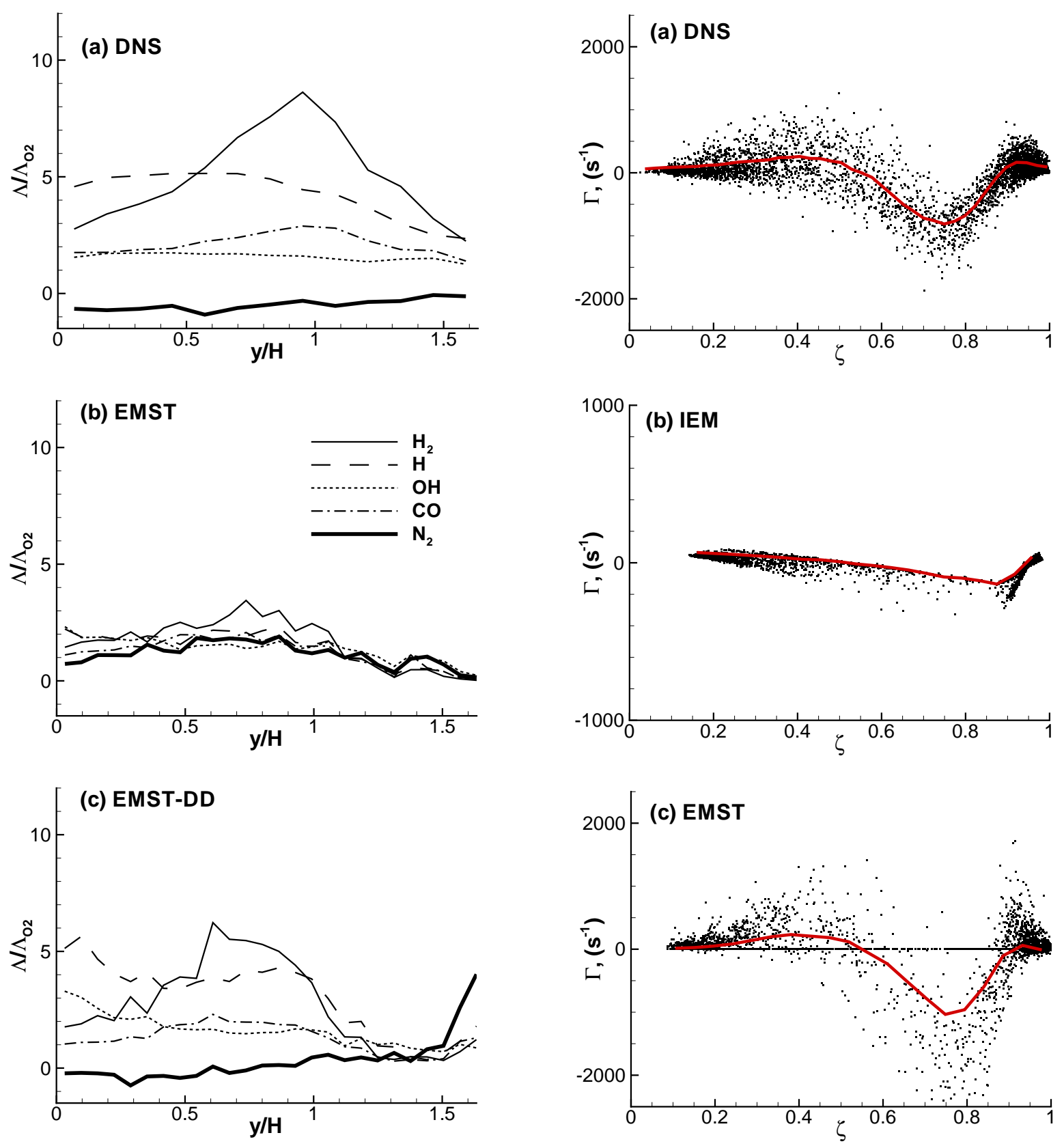

Figure 1: Ratios for $\Lambda$ between selected species $\left(\mathrm{H}_{2}, \mathrm{H}, \mathrm{OH}, \mathrm{CO}, \mathrm{N}_{2}\right)$ and $\mathrm{O}_{2}$ in the (a) DNS, (b) EMST, and (c) EMST-DD simulations.

Figure 2: Diffusion rates of $\mathrm{CO}$ in progress variable space from (a) the DNS, (b) IEM, and (c) EMST simulations. The solid line is the conditional mean. 
dictions of the conditional average $\mathrm{CO}$ and $\mathrm{O}_{2}$ mass fraction and diffusion rates in Fig. 3 are changed little by the differential diffusion correction, and they agree with the DNS data closely.

Variation of the $\mathrm{Y}_{\mathrm{N}_{2}}$ conditional mean mass fraction is shown in Fig. 3e. There is a finite variation of $\mathrm{Y}_{\mathrm{N}_{2}}$ in the initial condition of the PDF calculations, and this persists even in the case of the EMST model. The effect of differential diffusion is seen in the DNS and EMST-DD profiles, where differential diffusion moves $\mathrm{Y}_{\mathrm{N}_{2}}$ from the preheat-zone into the reactant zone, generating variance. While the variation of $\mathrm{Y}_{\mathrm{N}_{2}}$ is small, and unlikely to have any practical significance in a combustion system, we note that the EMST-DD model reproduces this feature which is symptomatic of differential diffusion. We note also that the EMST-DD reproduces the negative values, and the shape of the cross-stream variation, of the $\Lambda_{N_{2}} / \Lambda_{O_{2}}$ mixing ratio seen in the DNS in Fig. 1.

\section{Conclusions}

The performance of the EMST-DD, EMST, and IEM models has been assessed by comparison of conditional statistics and mixing timescales with DNS data for a premixed turbulent Bunsen flame. Unlike the IEM model, the EMST models describe the structure of mixing through the flame correctly. Combining the EMST description of scalar localness with differential mixing rates, the EMSTDD model, predicts mixing rate ratios similar to those in the DNS. The EMST-DD model predictions reproduce several features which characterize flamelet combustion with differential diffusion, including predicting that variance of $\mathrm{Y}_{\mathrm{N}_{2}}$ is produced in the premixed combustion DNS. These observations agree with, and provide further support for the conclusions of the main paper.

\section{References}

[1] R. Sankaran, E.R. Hawkes, C.S. Yoo, J.H. Chen, T. Lu, C.K. Law, in: 5th U.S. National Combustion Meeting, 2007 B09.

[2] E.S. Richardson, R. Sankaran, R.W. Grout, J.H. Chen, Combust. Flame 157 (3) (2010) 506-515.

[3] S.B. Pope, Turbulent Flows, Cambridge University Press, Cambridge, 2000, p.547.

[4] S. Subramaniam, S.B. Pope, Combust. Flame 115 (1998) 487-514. 

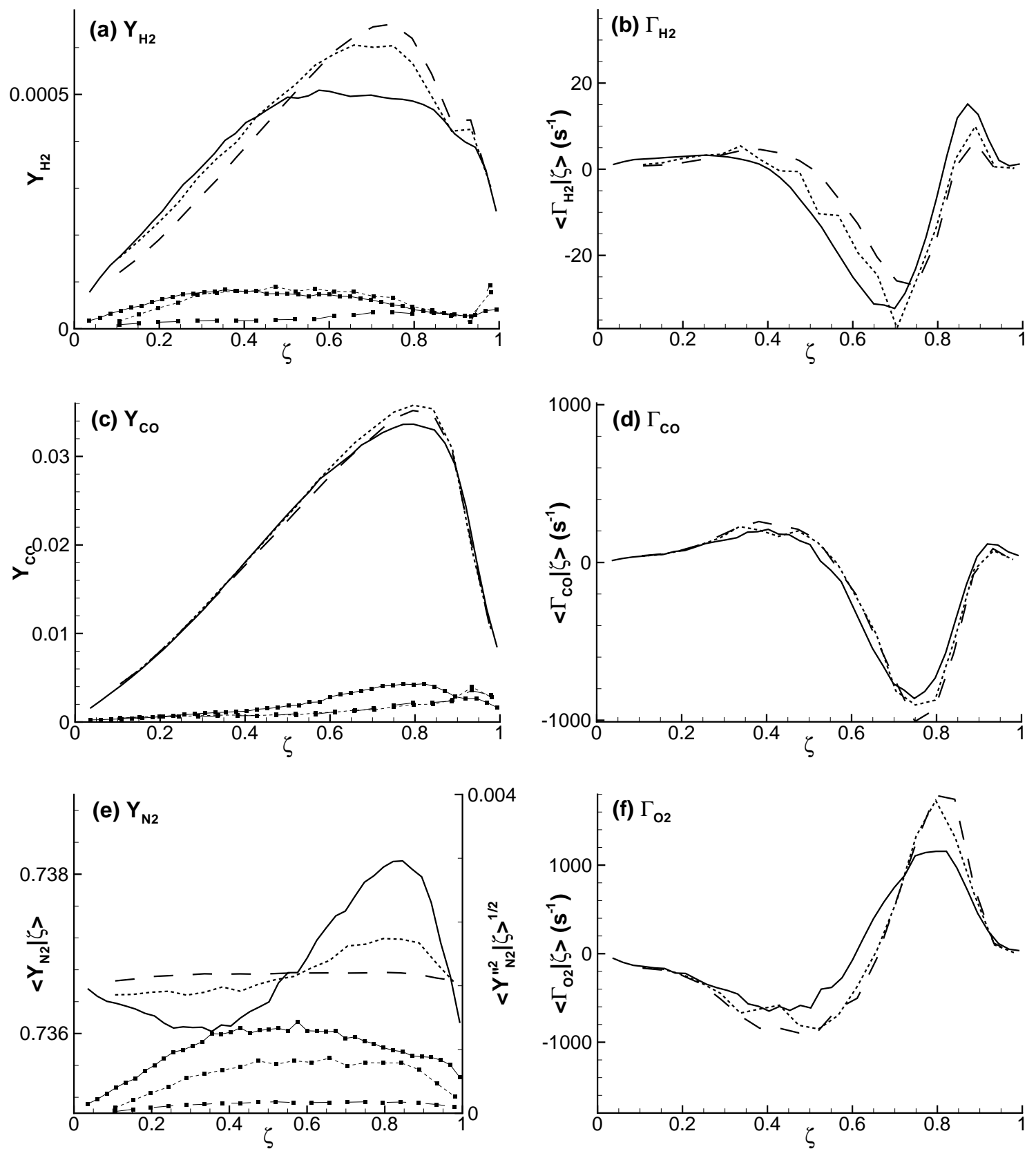

Figure 3: DNS (solid lines), EMST (dashes), and EMST-DD (dots) data for conditional mean (lines) and rms (lines with symbols) species mass fractions of $\mathrm{H}_{2}, \mathrm{CO}$, and $\mathrm{N}_{2}$ (left column), and diffusion rates of $\mathrm{H}_{2}$, $\mathrm{CO}$, and $\mathrm{O}_{2}$ (right column). 